\title{
How the Speed and Latitude of the Jet Stream Affect the Downstream Response to Recurving Tropical Cyclones
}

\author{
PETER M. FINOCCHIO \\ National Research Council, Monterey, California \\ JAMES D. DOYLE \\ Naval Research Laboratory, Monterey, California
}

(Manuscript received 22 February 2019, in final form 12 June 2019)

\begin{abstract}
Recurving tropical cyclones (TCs) that interact with the jet stream can trigger Rossby wave packets that amplify the flow far downstream, but the extent to which the jet stream modulates TC-jet interactions and the development of the downstream response remains unclear. This study uses 25 idealized simulations from the COAMPS-TC model to examine how the latitude and maximum wind speed of an initially zonal jet stream affect the downstream response to recurving TCs. During the first 5 days of the simulations, the formation of a jet streak and a ridge immediately downstream of the TC occurs earlier on low-latitude jets than on high-latitude jets. This is due to weaker TC inertial stability at low latitudes, which promotes negative potential vorticity advection by the irrotational outflow along the jet. Increasing the speed of the jet locally reduces inertial stability poleward of the TC, but does not profoundly affect the ability of the TC to perturb the jet. Beyond 5 days, the highest-latitude and fastest jets, which have the largest baroclinic growth rates, exhibit the highest-amplitude Rossby waves and the most rapidly intensifying surface cyclones farther downstream of the TCs. Both measures of the downstream response are more sensitive to changing the speed than the latitude of the jet. Deactivating condensational heating, shortly after TCs trigger a Rossby wave packet, decreases the amplitude and variability of the downstream flow by up to 3 times relative to the fully moist simulations. This result emphasizes the importance of moist diabatic processes for generating an amplified downstream response to recurving TCs within 7-10 days.
\end{abstract}

\section{Introduction}

Tropical cyclones (TCs) that recurve and undergo an extratropical transition (ET) can induce a near-hemispheric response that affects the weather and its predictability over large areas of the planet (see recent reviews by Evans et al. 2017; Keller et al. 2019). The downstream response is primarily accomplished through the initiation and subsequent propagation of Rossby wave packets along zonally extensive waveguides that are characteristic features of the midlatitude upper-tropospheric flow (Archambault et al. 2013, 2015; Quinting and Jones 2016; Wirth et al. 2018). Amplifying Rossby waves initiated during ET have been linked to atmospheric blocking and extreme weather far downstream (Grams and Blumer 2015; Bosart et al. 2017; Riboldi et al. 2019), as well as

Corresponding author: Peter M. Finocchio, peter.finocchio.ctr@ nrlmry.navy.mil decreased downstream predictability (Harr et al. 2008; Anwender et al. 2008; Grams et al. 2015; Aiyyer 2015; Quinting and Jones 2016; Torn 2017). Improved prediction of the downstream impacts of recurving TCs on medium-range to subseasonal time scales requires a deeper understanding of the initiation of Rossby waves along the jet stream and the amplification of these waves as they propagate downstream. This study uses a set of idealized, full-physics simulations in order to address how characteristics of the jet stream modulate each of these processes.

Diabatic heating and the development of an uppertropospheric, irrotational outflow are hypothesized to be key processes by which TCs initiate Rossby wave packets. As recurving TCs approach the jet stream, their outflow becomes increasingly poleward-directed (Komaromi and Doyle 2018), leading to stronger negative potential vorticity (PV) advection along the waveguide. A jet streak generally forms poleward of 
the TC in response to the intensifying PV gradient, while a ridge forms immediately downstream of the TC from the diabatic reduction of PV within the outflow anticyclone (Wu and Kurihara 1996; Riemer et al. 2008; Riemer and Jones 2010; Archambault et al. 2013; Grams et al. 2013a; Grams and Blumer 2015; Grams and Archambault 2016). From the perspective of the quasigeostrophic thickness tendency, the cyclonic circulation of the TC also contributes to the formation of the downstream ridge through the poleward advection of warm, moist air in the lower troposphere on the downstream side of the TC (Torn 2010; Riemer and Jones 2010). The formation of a downstream ridge signals the initiation of a Rossby wave packet-an absolute vorticity-conserving response to the ageostrophic divergent forcing of the TC's irrotational outflow impinging on the jet (Hodyss and Hendricks 2010). Prolonged interactions between the TC and the jet stream are thus thought to be particularly effective at triggering Rossby waves, with the TC behaving as "a long-lived local wave maker" (Riemer et al. 2008; Keller 2017).

Rossby wave energy propagation away from the TC is largely restricted to narrow corridors in the upper troposphere that are associated with the predominant jet streams. Several studies on global atmospheric teleconnections have established that the strong PV gradients in the upper troposphere refract Rossby wave energy toward the core of the jet stream, allowing the jet stream to function as a waveguide (Branstator 1983; Hoskins and Ambrizzi 1993; Hakim 2003; Martius et al. 2010). As trapped wave packets propagate along waveguides, baroclinic growth within the wave packet amplifies the downstream flow pattern through a process known as downstream cyclogenesis (Simmons and Hoskins 1979). The downstream cascade of wave energy has also been viewed as an ageostrophic flux of eddy kinetic energy away from the transitioning TC, which seeds the development of downstream troughs and ridges (Orlanski and Sheldon 1995; Harr and Dea 2009; Quinting and Jones 2016; Keller 2017).

Much of the recent interest in TC interactions with the midlatitude waveguide concerns the compromised downstream predictability that often follows such interactions. Harr et al. (2008) and Anwender et al. (2008) consistently found elevated uncertainty in the amplitude and phase of downstream atmospheric wave patterns after several ET events in the western North Pacific. More recent studies using ensemble reforecasts for large samples of ET cases find statistically significant increases in downstream ensemble spread and degraded skill in medium-range forecasts initialized just prior to ET (Aiyyer 2015; Grams et al. 2015; Quinting and Jones 2016; Torn 2017).
One source of uncertainty in the evolution of the downstream flow following ET is the phasing between the TC and an upstream trough. When a recurving TC becomes phase-locked with an approaching trough, a resonant interaction takes place in which the TC and trough mutually amplify (Ritchie and Elsberry 2003, 2007; Hodyss and Hendricks 2010). It has been argued that such resonant interactions increase the probability of a significant downstream response (Grams et al. 2013b; Aiyyer 2015; Grams et al. 2015; Riboldi et al. 2019). Whether phase-locking occurs, however, is sensitive to subtle changes in the amplitude and position of the upstream trough relative to the recurving TC (Riemer and Jones 2010; Scheck et al. 2011; Grams et al. 2013b; Riemer and Jones 2014; Keller 2017) and such sensitivities are known to contribute to the reduced downstream predictability in the days following recurvature (Reynolds et al. 2009). Torn and Hakim (2015) pointed out that the reduced predictability following ET might also be a result of ET generating new waves rather than amplifying existing waves that are sampled by upstream observations. Moreover, Torn (2017) demonstrated that the elevated forecast spread emanates downstream from a transitioning TC regardless of whether there is an upstream wave packet. Based on these results, he reasoned that uncertainties associated with wave packet initiation are likely to be an additional source of downstream forecast errors.

Diabatic heating in mesoscale convective systems occasionally causes large forecast errors far downstream (Rodwell et al. 2013; Martínez-Alvarado et al. 2016), and may be a key physical process underlying the uncertainty associated with Rossby wave initiation from TCs. Pantillon et al. (2013) found that model errors associated with the diabatic outflow structure of Atlantic Hurricane Helene (2006) propagated downstream at the group velocity of a Rossby wave packet, eventually affecting forecasts of explosive cyclogenesis over Europe. Moisture fluxes and condensation in ascending airstreams along the baroclinic zone to the east of the transitioning TC are additional sources of uncertainty in downstream flow amplification (Torn 2010; Grams et al. 2011; Grams and Archambault 2016). Therefore, even in the absence of phasing uncertainties, there remains considerable uncertainty associated with moist diabatic processes near the TC.

Given the potential importance of the TC in initiating Rossby wave packets along waveguides, some studies have sought to find a systematic relationship between the amplitude of the downstream response and the size or intensity of TCs. Much of this work is motivated by theoretical and modeling studies that demonstrate the sensitivity of remote responses to the characteristics 
of a TC-like divergent forcing located in the upper troposphere (Hoskins and Ambrizzi 1993; Schwierz et al. 2004). Riemer et al. (2008) conducted idealized simulations that systematically changed the size and intensity of the transitioning TC and found the downstream ridge to be less amplified for smaller and weaker TCs. In a follow-up study, they found that larger and more intense TCs produced a somewhat more amplified upper-level wave train (Riemer and Jones 2010). However, mounting evidence from climatological analysis of recurving TCs suggests that the size and intensity of the TC at the time of recurvature does not consistently determine the nature of the downstream response (Archambault et al. 2013; Quinting and Jones 2016; Riboldi et al. 2018). Reynolds et al. (2009) found that the growth of singular vector perturbations in a sample of recurving typhoons in the western North Pacific exhibited a clearer relationship to the baroclinic instability across the North Pacific than to the storm intensity at the beginning of the growth interval.

Only a few studies have explored how the structure or latitude of the jet stream affects the downstream response to recurving TCs, and no clear consensus has emerged. The additional sensitivity tests that Riemer et al. (2008) conducted in which they changed the maximum speed of the jet demonstrated that weaker jets become wavier following ET and produce more slowly developing downstream cyclones. However, the small number of experiments was not sufficient to characterize the sensitivity of the downstream response to the speed of the jet stream. Riboldi et al. (2018) found that Rossby wave initiation is more likely to occur in the presence of weaker jets because stronger jets limit ridge building by advecting PV anomalies produced during the TC interaction too rapidly downstream. However, Manola et al. (2013) found stronger and narrower jets to be more effective at ducting perturbation Rossby wave energy downstream in a dry barotropic vorticity model, which is consistent with theoretical predictions of Hoskins and Ambrizzi (1993). The divergent wave source in their simulations also produced a more amplified downstream response if both the source and the jet were shifted poleward.

The goal of this study is to use a set of idealized simulations to understand the extent to which the strength and latitude of a zonal jet in the upper troposphere influence the downstream response to recurving TCs and the variability in this response. To more easily attribute the downstream response to systematic changes to the initial structure of the jet, we initialize zonal jets without baroclinic waves. This is not an entirely unrealistic simplification, as Riboldi et al. (2018) found $23 \%$ of recurving TCs in the western North Pacific interacted with a primarily zonal jet. All simulations utilize a nonhydrostatic mesoscale model described in section 2 . Results on how the jet modulates TC-jet interactions and the amplitude and variability of the downstream response appear in section 3. Section 4 describes a set of restart simulations in which condensational heating is deactivated in the model in order to explicitly examine how moist diabatic processes affect the downstream response. Finally, section 5 contains a discussion of the results and conclusions.

\section{Model description and experimental design}

For all simulations in this study, we use version 5 of the Coupled Ocean-Atmosphere Mesoscale Prediction System for Tropical Cyclones (COAMPS-TC; Hodur 1997; Chen et al. 2003; Doyle et al. 2014). COAMPS-TC solves the compressible, nonhydrostatic equations of motion and utilizes a terrain-following sigma-height coordinate system. For simplicity, we only use the atmospheric component of COAMPS-TC without coupling to an ocean model. The model domain is a large aquachannel with periodic boundaries in the $x$ direction and closed boundaries in the $y$ direction. The horizontal resolution is $15 \mathrm{~km}$ and the dimensions of the domain are $15000 \mathrm{~km} \times 7500 \mathrm{~km}$, spanning latitudes from $6^{\circ}$ to $71^{\circ} \mathrm{N}$ and the approximate longitudinal extent of the Pacific Ocean. The Coriolis parameter varies meridionally with Earth's geometry (i.e., $f=2 \Omega \sin \phi$, where $\phi$ is latitude and $\Omega$ is Earth's rotational frequency). In the vertical, we use 40 irregularly spaced sigma mass levels extending up to an altitude of $29 \mathrm{~km}$ $(15 \mathrm{hPa})$. The vertical grid spacing is less than $250 \mathrm{~m}$ within the boundary layer and increases to around $1 \mathrm{~km}$ near the tropopause. The parameterized physics include a 6-class single-moment microphysics scheme based on a modified Rutledge and Hobbs (1983) and Lin et al. (1983) scheme, a shallow cumulus scheme paired with the Kain-Fritsch cumulus scheme (Kain 2004), and the subgrid-scale mixing scheme described in Hodur (1997) with dissipative heating based on Jin et al. (2007) and a mixing length adjustment suitable for deep convection in TCs (Bougeault 1985). No radiation schemes are used.

The initial conditions consist of a westerly jet and a TC vortex in an otherwise quiescent environment that has the moist tropical thermodynamic sounding of Dunion (2011). Figure 1 illustrates the horizontal and vertical cross sections through the jet and the TC. The westerly jet extends across the entire domain and is designed to be similar to the jet used in the "SR" experiment of Riemer et al. (2008). Unlike Riemer et al. (2008) who prescribe the mass field and obtain the balanced winds, we prescribe the wind field and obtain the mass fields through an iterative thermal wind balancing procedure. The environment to the 

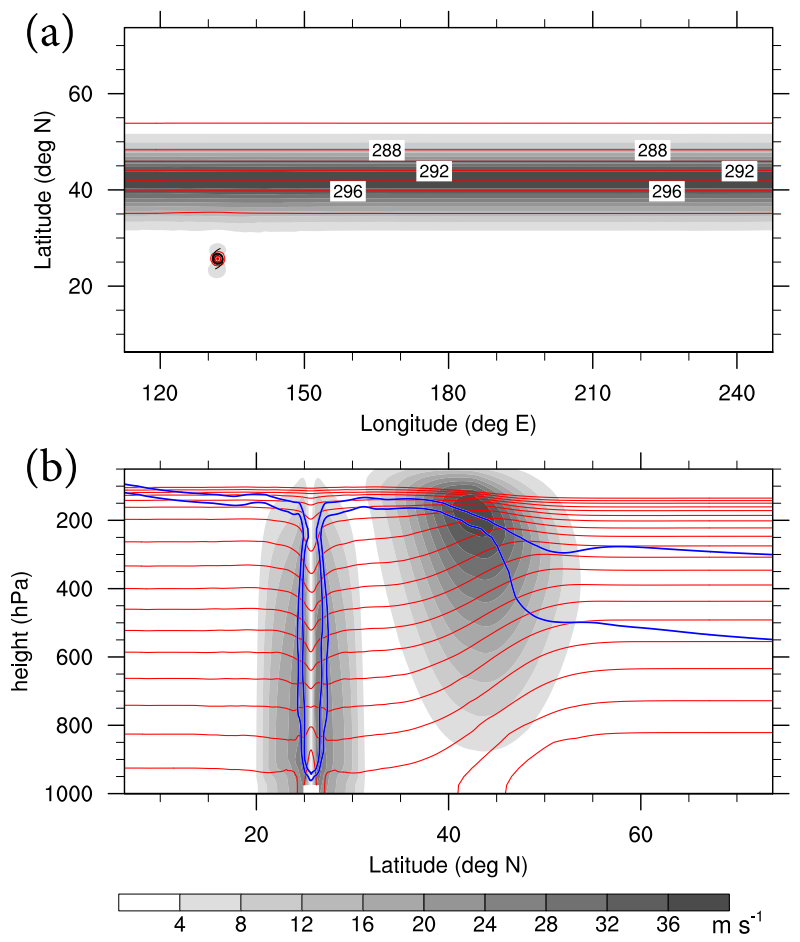

FIG. 1. (a) Horizontal cross section of total wind speed at the jet maximum level of $175 \mathrm{hPa}$ (shading, $\mathrm{m} \mathrm{s}^{-1}$ ) and surface potential temperature (red contours, K) at $t=6 \mathrm{~h}$ for the $40 \mathrm{~m} \mathrm{~s}^{-1}$ jet at $42.5^{\circ} \mathrm{N}$. (b) Vertical cross section at the same time through the TC and jet along the longitude denoted by the black dashed line in (a). Shading is total wind speed $\left(\mathrm{m} \mathrm{s}^{-1}\right)$, red contours are potential temperature (from 290 to $380 \mathrm{~K}$, every $5 \mathrm{~K}$ ), and blue contours are the 1- and 2-PVU contours.

south of the jet retains the moist tropical sounding after thermal wind balancing while the environment north of the jet becomes more representative of the midlatitudes. The sea surface temperature (SST) is fixed at $29^{\circ} \mathrm{C}$ south of the jet and at $1^{\circ} \mathrm{C}$ warmer than the initial surface air temperature along and north of the jet axis after the mass fields are brought into thermal wind balance with the jet.

The prescribed wind field of the jet decays as a Gaussian to the north and south of the jet axis, and nearly as a Gaussian above and below the jet axis according to

$u_{\text {jet }}=u_{\max } \times \exp \left[-\frac{\left(j-j_{\max }\right)^{2}}{\left(j_{\text {width }} / 2\right)^{2}}\right] \times \exp \left[-\frac{\left(k-k_{\max }\right)^{3 / 2}}{\left(k_{\text {width }} / 2\right)^{3 / 2}}\right]$,

where $u_{\max }$ is the maximum wind speed; $j$ and $k$ are the gridpoint indices in the $y$ and $z$ directions, respectively; $j_{\max }$ is the $y$ index of the jet axis; $k_{\max }$ is the sigma level index of the jet axis; and $j_{\text {width }}$ and $k_{\text {width }}$ are the $e$-folding width and depth, respectively. The value of $k_{\max }$ is set such that the westerly winds of the jet are maximized at about $175 \mathrm{hPa}$, as in Riemer et al. (2008). The value of

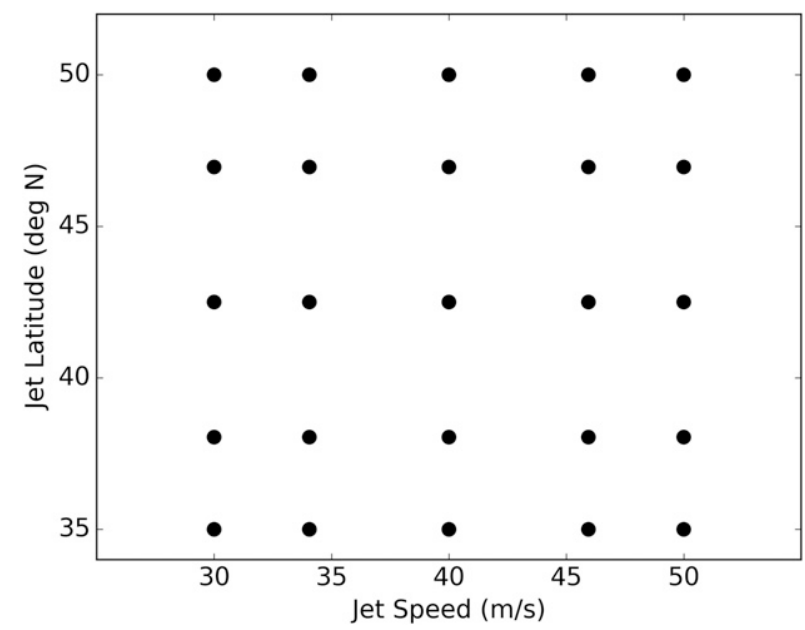

FIG. 2. Grid of latitude and maximum wind speeds used to initialize the jets in each of the 25 simulations.

$j_{\text {width }}$ is set such that the jet has a meridional extent of $1650 \mathrm{~km}$. To obtain the slight asymmetry in the $y$ direction about the jet axis evident in Fig. $1 b, k_{\max }$ is made to be a function of latitude, with a gradual increase in the height of the jet axis to the south and a steeper decrease in the height of the jet axis to the north of the jet.

The two free parameters for prescribing the structure of the jet are the latitude of the jet axis, which determines $j_{\max }$, and the jet maximum wind speed $u_{\max }$. We conduct 25 simulations using different combinations of jet latitude and speed $\left(u_{\max }\right)$. Each point in Fig. 2 corresponds to the combination of jet latitude and speed that is used to initialize a simulation. The jet latitudes tested in this study range from $35^{\circ}$ to $50^{\circ} \mathrm{N}^{1}$ and the jet maximum wind speeds range from 30 to $50 \mathrm{~m} \mathrm{~s}^{-1}$. These ranges are based on frequently observed jet maximum wind speeds and latitudes during the summer months (Manola et al. 2013). The two-dimensional parameter space is sampled according to Gauss quadrature rules. The purpose of using Gauss quadrature is to optimally fit two-dimensional polynomial series to the model output in order to obtain smooth response surfaces within the jet latitude-speed input space depicted in Fig. 2 (analysis

${ }^{1}$ At $50^{\circ} \mathrm{N}$, the jet wind speed maximum altitude of $175 \mathrm{hPa}$ may be unrealistically high. In test simulations with the jet maximum lowered to $220 \mathrm{hPa}$, the amplitude of the downstream response to the recurving TCs after several days is qualitatively similar to the simulations with the jet at $175 \mathrm{hPa}$. However, lowering the height of the jet maximum also increases the baroclinicity of the jet and decreases the static stability south of the jet, causing stronger TCs and downstream surface cyclones to form and changing some details of the downstream response. Analyzing the sensitivity of the downstream response to the initial altitude of the jet is beyond the scope of this study. 
of these response surfaces appears in section 3b). By sampling five points in each dimension of the parameter space with the 25 simulations, we are able to fit a fourthorder polynomial series to the model output.

In each simulation, a TC vortex is inserted $17^{\circ}$ to the south of the jet axis. The initial $17^{\circ}$ spacing between the $\mathrm{TC}$ and the jet axis was chosen to be large enough that the TCs can intensify and develop axisymmetric moist convection in a low-shear environment over the first $48 \mathrm{~h}$ of the simulations, but not so large that the closed southern boundary of the channel domain restricts the divergent outflow of TCs in the low-latitude jet simulations. The TC is a modified Rankine vortex with a maximum tangential wind speed of $40 \mathrm{~m} \mathrm{~s}^{-1}$ at a radius of $75 \mathrm{~km}$ and an altitude of $800 \mathrm{~m}$. The tangential winds decay exponentially above and below $800 \mathrm{~m}$. Within the region containing the cyclonic winds of the $\mathrm{TC}$, the mass fields are brought into gradient wind balance with the vortex wind field through an iterative procedure described in Nolan et al. (2001). Each simulation is implemented for $240 \mathrm{~h}$ in order to allow time for the TC to intensify, recurve, and generate a downstream response.

The $15-\mathrm{km}$ horizontal resolution of the model is too coarse to capture the detailed structure and intensity evolution of the TC. Therefore, we conducted an additional test simulation for the $40 \mathrm{~m} \mathrm{~s}^{-1}$ jet at $42.5^{\circ} \mathrm{N}$ using $5-\mathrm{km}$ horizontal resolution and no parameterization for deep convection (not shown). While the TC remains stronger in the high-resolution simulation, the downstream response to the TC is qualitatively similar to the corresponding $15-\mathrm{km}$ simulation. Four additional $15-\mathrm{km}$ simulations were also implemented for the fastest and weakest jets at $35^{\circ}$ and $50^{\circ} \mathrm{N}$ without a TC in order to determine the stability of these jets (not shown). Shortly after 5 days, cyclonic disturbances along the jet axis begin appear near the surface and steadily intensify through $240 \mathrm{~h}$. These disturbances originate from moist convection in the most baroclinically unstable band along the jet axis. Despite these disturbances, the jets retain their zonally averaged structure through $240 \mathrm{~h}$ and lack the large-scale Rossby waves that form in the simulations with TCs. Therefore, we are reasonably confident that the basic results of this study are not critically affected by the relatively coarse horizontal resolution or the disturbances that form along the jet in the absence of a TC.

\section{Results}

\section{a. TC-jet interactions}

In this section, we discuss how changing the latitude or the maximum wind speed of the idealized jet affects the TCs and their interactions with the jet. Figure 3 shows
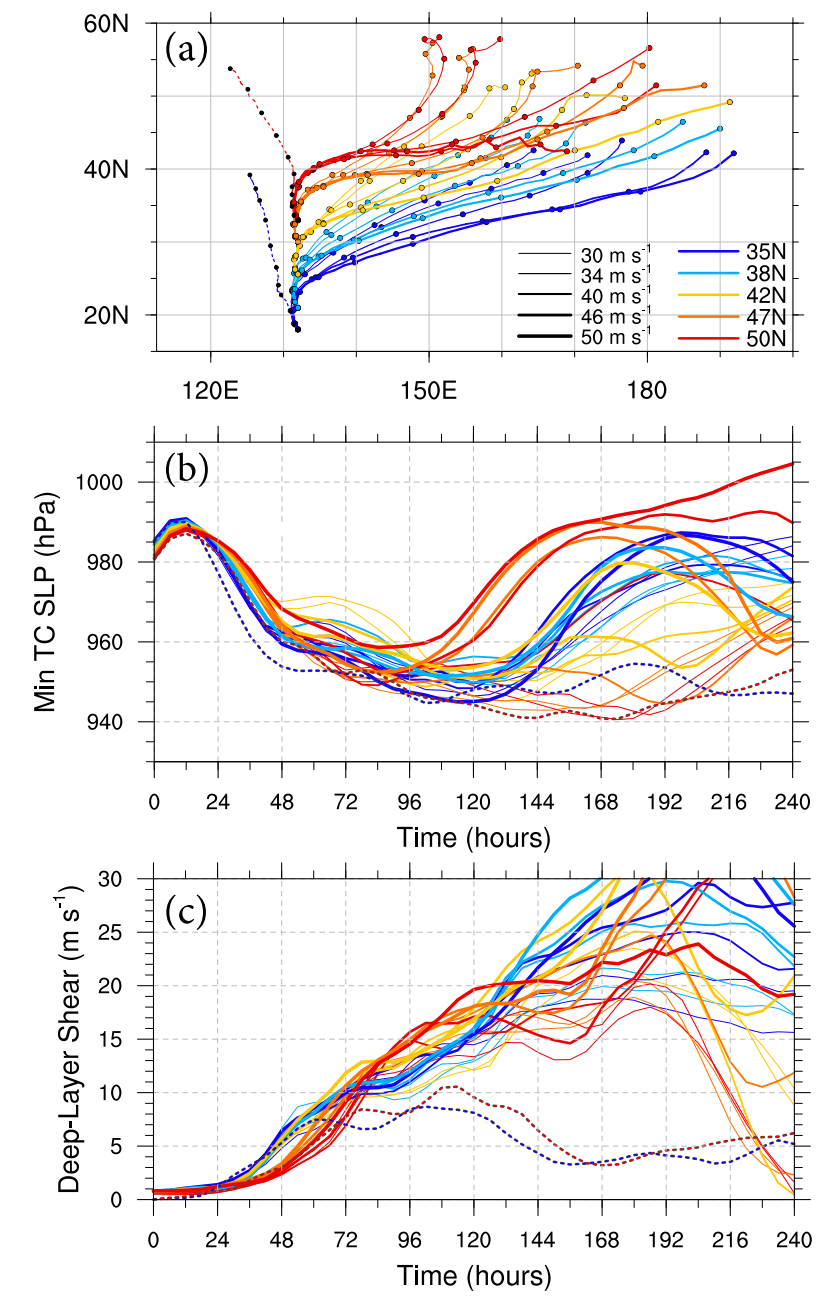

FIG. 3. (a) TC tracks for the 25 jet simulations (solid lines) and two simulations without a jet (blue and red dashed lines). Thinner (thicker) lines correspond to weaker (stronger) initial jets, and line colors denote latitude (see legend). Dots are drawn every $24 \mathrm{~h}$ from 0 to $240 \mathrm{~h}$. (b) Time series of TC minimum sea level pressure $(\mathrm{hPa})$ for the 25 jet simulations and 2 simulations without a jet. (c) Time series of the $200-850-\mathrm{hPa}$ vertical wind shear averaged within $200-800 \mathrm{~km}$ from the TC center position. Line colors, thicknesses, and styles in (b) and (c) correspond to TC tracks in (a).

the TC tracks (Fig. 3a), and time series of TC intensity (in terms of minimum sea level pressure, Fig. 3b) and 200-850-hPa vertical wind shear averaged between 200 and $800 \mathrm{~km}$ from the TC centers (Fig. 3c) for the 25 different jet simulations. The dashed lines correspond to two additional simulations without a jet for the lowestand highest-latitude TCs. TC positions in Fig. 3a correspond to the location of minimum sea level pressure. All of the TCs move toward the north-northwest during the first $48 \mathrm{~h}$ due to beta drift (Fiorino and Elsberry 1989; Wang and Holland 1996) and exhibit an increase in vertical wind shear (Fig. 3c) associated with the development 

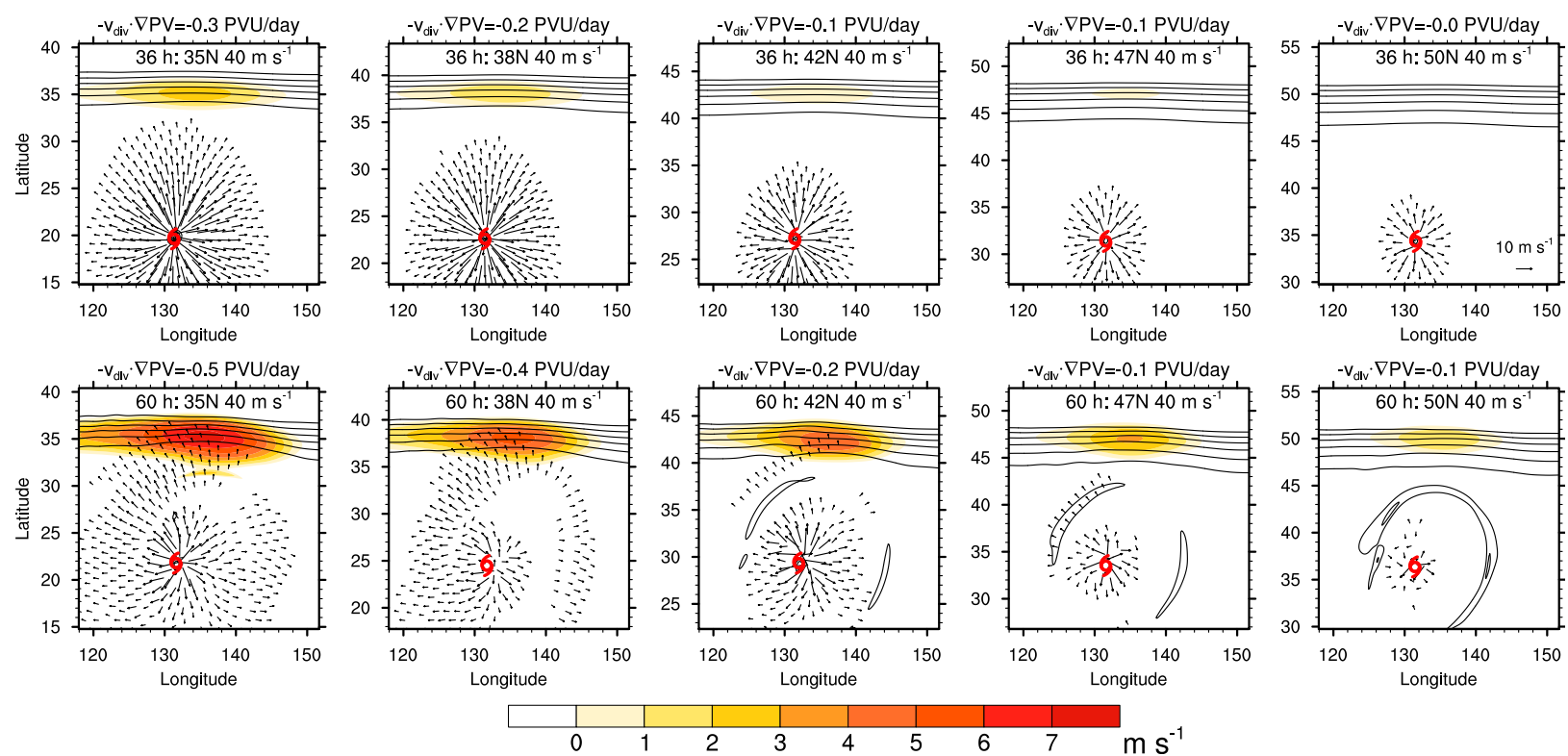

FIG. 4. Irrotational winds exceeding $2.5 \mathrm{~m} \mathrm{~s}^{-1}$ (vectors), nondivergent winds in excess of the initial values (shading), and potential vorticity (black contours from 1.5 to 4 PVU every 0.5 PVU) for the five simulations with $40 \mathrm{~m} \mathrm{~s}^{-1}$ jets at (top) $t=36 \mathrm{~h}$ and (bottom) $t=60 \mathrm{~h}$. All quantities are vertically averaged between 150 and $200 \mathrm{hPa}$. The red symbol denotes the TC position. Printed atop each panel is the instantaneous value of the PV advection along the jet by the irrotational wind averaged between 150 and $200 \mathrm{hPa}$.

of asymmetric outflow and upper-level beta gyres (Ritchie and Frank 2007). Between 48 and 120 h, TCs in jet environments accelerate toward the northeast as they approach the jets (Fig. 3a) and the vertical wind shear continues to steadily increase (Fig. 3c). The TC tracks diverge during the last 5 days of the simulations; TCs approaching the strongest jets tend to continue moving toward the northeast while those approaching the weaker jets move more toward the north. For the intensity (Fig. 3b), the TCs spin down during the first $12 \mathrm{~h}$ and then rapidly intensify to approximately $965 \mathrm{hPa}$ by $48 \mathrm{~h}$. Intensification slows between 48 and $84 \mathrm{~h}$, even for the simulations without a jet, suggesting that the slower intensification rate may be due to the increasing beta shear rather than the increasing shear or decreasing SST associated with the jets. However, by $96 \mathrm{~h}$, the TCs approaching the strongest jets do begin to weaken as a result of vertical wind shear that exceeds the shear in the simulations without a jet (Fig. 3c). The TCs approaching weaker jets maintain minimum central pressures less than $980 \mathrm{hPa}$. Some even slowly intensify to near $940 \mathrm{hPa}$ through $180 \mathrm{~h}$, like the TCs in simulations without jets. Beyond $180 \mathrm{~h}$, some of the TCs that weakened in the strong jets undergo an extratropical reintensification. We will not discuss this reintensification in detail because it occurs several days after the triggering of Rossby wave packets that affect the downstream response.

Previous studies have hypothesized that the degree of interaction between the $\mathrm{TC}$ and the jet stream determines the extent of downstream flow amplification that follows (e.g., Archambault et al. 2013, 2015). Therefore, most of this section will focus on how the jet modulates these TC-extratropical flow interactions during the first $120 \mathrm{~h}$. Our discussion will be framed around two key components of Rossby wave excitation: the acceleration of a jet streak and the development of a ridge immediately downstream of the TC.

Local acceleration of a jet streak to the north of a recurving TC occurs when the TC outflow advects lowPV air toward the strong PV gradient of the jet. All of our simulations exhibit a period of jet streak formation, but the degree and timing varies considerably among the simulations. We find that the latitude of the jet is a better determinant of the rate of jet streak formation than the maximum wind speed of the jet. To illustrate this, Fig. 4 depicts snapshots at 36 and $60 \mathrm{~h}$ of the irrotational winds exceeding $2.5 \mathrm{~m} \mathrm{~s}^{-1}$ (vectors) and nondivergent winds in excess of the values at $t=0 \mathrm{~h}$ (shading) vertically averaged from 150 to $200 \mathrm{hPa}$ for the five simulations with $40 \mathrm{~m} \mathrm{~s}^{-1}$ jets. The winds are obtained from a Helmholtz decomposition of the upper-level wind field. At $36 \mathrm{~h}$, differences in the extent of the TC outflow are clearly apparent at the different latitudes, where irrotational winds exceeding $2.5 \mathrm{~m} \mathrm{~s}^{-1}$ in the lowest-latitude simulation extend farther from the TC center than in the highest-latitude simulation. Also evident at this time is a nascent jet streak forming to the north of the TC in the lower-latitude jet simulations. By $60 \mathrm{~h}$, the jet streak is 

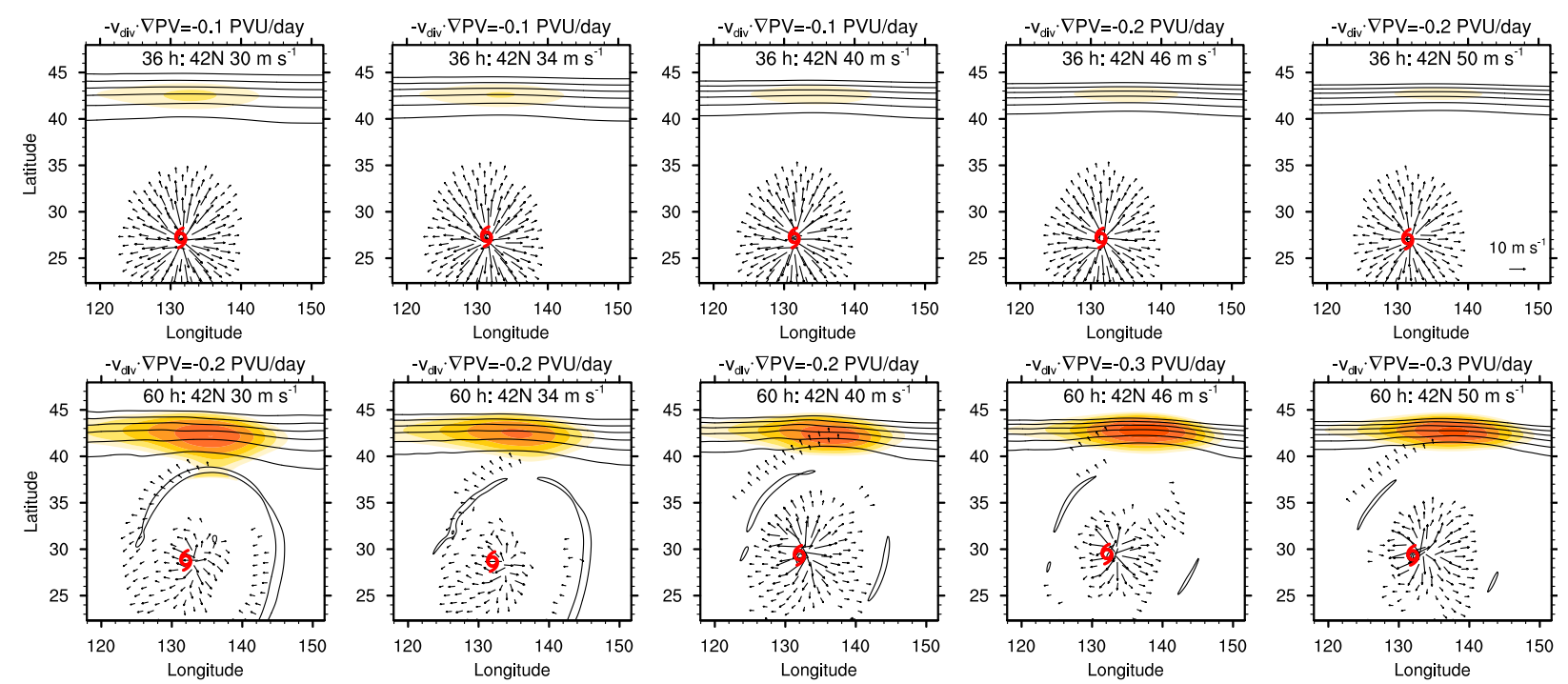

FIG. 5. As in Fig. 4, but for the five simulations with jets initially at $42.5^{\circ} \mathrm{N}$.

up to $5 \mathrm{~m} \mathrm{~s}^{-1}$ stronger on the low-latitude jets because of the more prolonged interaction of the broader outflow from the TC with the jet. Figure 5 depicts the same fields for five simulations with jets initially at $42.5^{\circ} \mathrm{N}$. At $36 \mathrm{~h}$, the outflow vectors extend a similar distance from the TC approaching the $30 \mathrm{~m} \mathrm{~s}^{-1}$ jet as they do from the TC approaching the $50 \mathrm{~m} \mathrm{~s}^{-1}$ jet. By $60 \mathrm{~h}$, the magnitude of the jet streak northeast of the recurving TCs is $1-2 \mathrm{~m} \mathrm{~s}^{-1}$ stronger for the faster jets compared to the $30 \mathrm{~m} \mathrm{~s}^{-1}$ jet.

The broader irrotational outflow of a low-latitude TC is a consequence of lower inertial stability in the TC environment. Inertial stability measures the resistance to radial motion and is defined in a TC-relative cylindrical coordinate system as $I^{2}=\left(f+2 v_{t} / r\right)(f+\zeta)$, where $v_{t}$ is the tangential wind, $r$ is the radius from the TC center, and $\zeta$ is the vertical relative vorticity. In our simulations, inertial stability in the TC environment is reduced by either decreasing the initial latitude of the jet, which decreases the Coriolis parameter $f$, or by increasing the speed of the jet, which increases the anticyclonic (negative) shear vorticity south of the jet axis. It is important to note that increasing the speed of the jet locally reduces inertial stability in the region north of the $\mathrm{TC}$, while decreasing the latitude of the TC and the jet reduces inertial stability throughout the TC environment. The difference between globally versus locally reducing TC inertial stability likely explains why jet streak formation is more sensitive to changing the latitude rather than the speed of the jet.

To further illustrate this, Fig. 6 shows time series of TC environmental inertial stability (Fig. 6a) and radial winds (Fig. 6b) averaged vertically from 100 to $200 \mathrm{hPa}$ and horizontally within the northern semicircle of the TCs between 500 and $1000 \mathrm{~km}$ from the TC center. Figure $6 \mathrm{c}$ is a corresponding time series of the PV advection by the irrotational wind in the 150-200-hPa layer and averaged in a box to the north of the TCs - similar to the TC-extratropical flow interaction metric defined in Archambault et al. (2013). The vertical averaging layers for this analysis are chosen to encompass the levels at which the jet winds and TC outflow are maximized in our simulations. Throughout the first two days of the simulations, differences in inertial stability due to jet latitude are larger than those due to jet speed (Fig. 6a). The average inertial stability in a simulation with a jet initialized at $35^{\circ} \mathrm{N}$ is up to $65 \%$ weaker than in a simulation with a jet initialized at $50^{\circ} \mathrm{N}$, while the inertial stability in a $50 \mathrm{~m} \mathrm{~s}^{-1}$ jet simulation is only up to $15 \%$ weaker than in a $30 \mathrm{~m} \mathrm{~s}^{-1}$ jet simulation. As a result, the strength of the radial outflow that develops to the north of the TCs from 24 to $36 \mathrm{~h}$ is primarily a function of jet latitude (Fig. 6b), with the globally reduced inertial stability at lower latitudes promoting stronger radial outflow, stronger negative PV advection (Fig. 6c), and the formation of a stronger jet streak (Fig. 4). From 72 to $120 \mathrm{~h}$, the ability of faster jets to reduce inertial stability becomes more apparent as the TCs approach the anticyclonic shear south of the jet (thick lines in Fig. 6a). Consequently, the TCs begin to exhibit stronger interactions with faster jets (thick lines in Figs. 6b,c).

Following the acceleration of a jet streak, upper-level PV anomalies emerge along the jet that signal the development of a ridge immediately downstream of the 

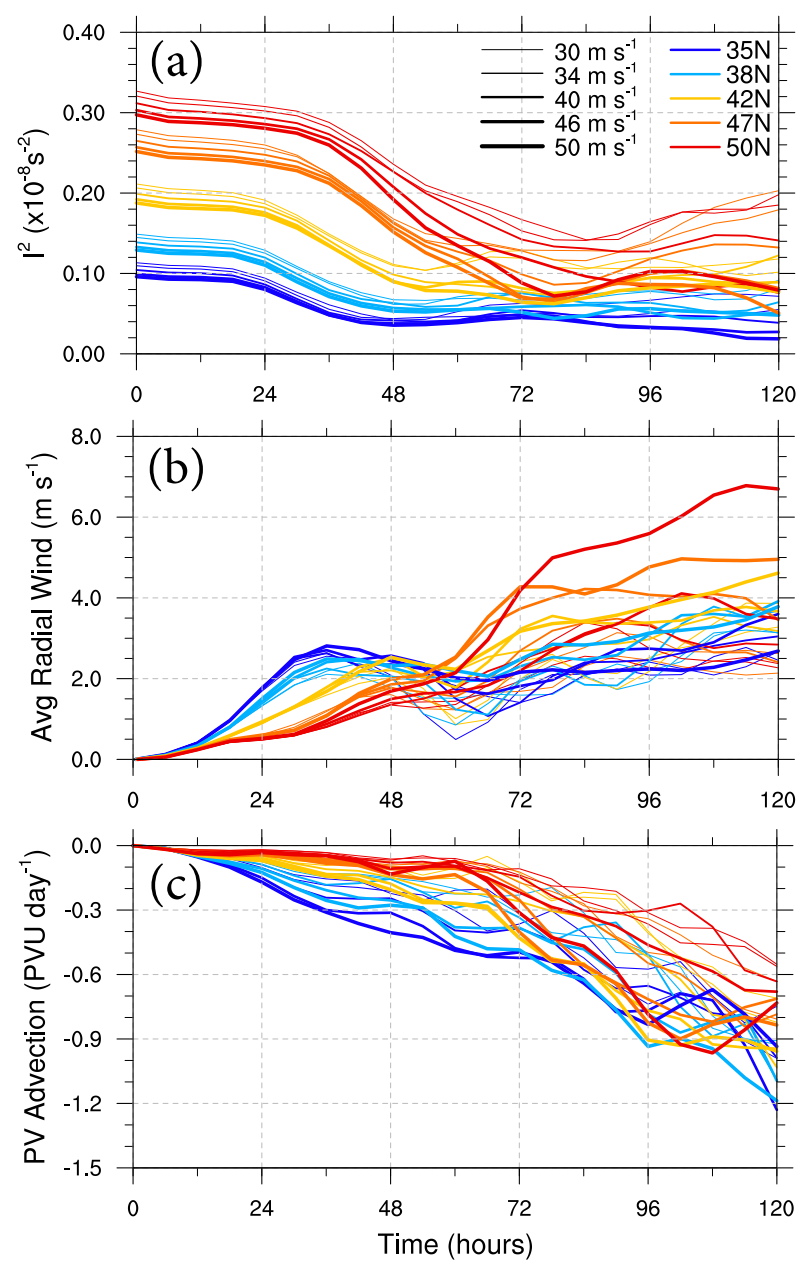

FIG. 6. Time series from 0 to $120 \mathrm{~h}$ of (a) TC-relative inertial stability averaged vertically from 100 to $200 \mathrm{hPa}$ and horizontally in the northern semicircle between 500 and $1000 \mathrm{~km}$ from the TC centers, (b) radial winds (positive $=$ outflow) averaged in the same region as inertial stability, and (c) potential vorticity advection by the irrotational wind averaged vertically from 150 to $200 \mathrm{hPa}$ and horizontally within a rectangular region extending $600 \mathrm{~km}$ south of the initial jet axis, $750 \mathrm{~km}$ north of the initial jet axis, $1800 \mathrm{~km}$ east of the TC, and $600 \mathrm{~km}$ west of the TC. Line colors and thicknesses are as in Fig. 3.

TC and the initiation of a Rossby wave packet. The formation of a negative PV anomaly associated with the primary downstream ridge has been attributed to the diabatic reduction of PV above condensational heating in convection and to the development of an outflow anticyclone that becomes displaced slightly downstream of recurving TCs (Wu and Kurihara 1996). Figures 7 and 8 show the $345-\mathrm{K}$ isentropic $\mathrm{PV}$ anomalies relative to the initial time $(t=0 \mathrm{~h})$ at 72 - and 120 -h simulation times, respectively. The left column in each figure depicts the five simulations with $40 \mathrm{~m} \mathrm{~s}^{-1}$ jets and the right column depicts the five simulations with jets initialized at $42.5^{\circ} \mathrm{N}$.
Because stronger and higher-latitude jets have stronger meridional PV gradients than weaker and lower-latitude jets, the magnitude of a PV anomaly will be larger for the same displacements of a PV contour from its initial position on stronger or higher-latitude jets. To compensate for this when comparing PV anomalies among the different jets, we multiply PV anomalies by a scaling factor equal to $\max (\Delta \mathrm{PV}) / \Delta \mathrm{PV}$, where $\Delta \mathrm{PV}$ is the meridional difference in $345-\mathrm{K}$ isentropic $\mathrm{PV}$ across a band extending from $150 \mathrm{~km}$ south to $150 \mathrm{~km}$ north of the initial jet axis, and $\max (\Delta \mathrm{PV})$ is the maximum of this PV difference over all 25 simulations. This scaling factor amplifies the PV anomaly by an amount that is proportional to how much weaker the initial PV gradient is than the simulation with the strongest meridional PV gradient (the $50 \mathrm{~m} \mathrm{~s}^{-1}$ jet at $50^{\circ} \mathrm{N}$ ). ${ }^{2}$

The PV dipole anomaly patterns that emerge by $72 \mathrm{~h}$ (Fig. 7) consist of a negative anomaly north of the TC that is associated with the primary downstream ridge, and a positive anomaly just downstream of the negative anomaly that is associated with the primary downstream trough. The magnitude of both the positive and negative upper-level PV anomalies at $72 \mathrm{~h}$ is up to 3 times larger along the jet at $35^{\circ} \mathrm{N}$ compared to the jet at $50^{\circ} \mathrm{N}$ (left column of Fig. 7). By contrast, the PV anomalies at $72 \mathrm{~h}$ are less sensitive to changing the speed of the jet (right column of Fig. 7); the magnitude of the negative (ridge) anomaly is only about $25 \%$ stronger on the $50 \mathrm{~m} \mathrm{~s}^{-1}$ jet than on the $30 \mathrm{~m} \mathrm{~s}^{-1}$ jet, and the differences in the magnitude of the positive (trough) anomalies among the different jet speed simulations are indistinguishably small at $72 \mathrm{~h}$, although PV anomalies greater than $1 \mathrm{PV}$ unit (PVU; 1 PVU $=10^{-6} \mathrm{~K} \mathrm{~kg}^{-1} \mathrm{~m}^{2} \mathrm{~s}^{-1}$ ) cover a larger area along the faster jets. By $120 \mathrm{~h}$ (Fig. 8), the magnitude of both the positive and negative anomalies is about twice as large on the jet at $35^{\circ} \mathrm{N}$ compared to the jet at $50^{\circ} \mathrm{N}$. The anomalies also begin to exhibit greater sensitivity to jet speed at this time, but are still only $20 \%-25 \%$ stronger in magnitude for the $50 \mathrm{~m} \mathrm{~s}^{-1}$ jet compared to the $30 \mathrm{~m} \mathrm{~s}^{-1}$ jet. Notably, the PV anomalies extend farther downstream along faster jets due to the stronger eastward advection of the anomalies. In general, the greater sensitivity of the PV anomalies to jet latitude than to jet speed reflects the global reduction in inertial stability brought about by decreasing the latitude of the TC and the jet, which promotes earlier and stronger TC-jet interactions. How the differences in the strength of TC-jet interactions among the simulations translate into differences in the response farther

\footnotetext{
${ }^{2}$ The largest scale factor among the jets depicted in Figs. 7 and 8 is 2.34 for the $30 \mathrm{~m} \mathrm{~s}^{-1}$ jet at $42.5^{\circ} \mathrm{N}$.
} 

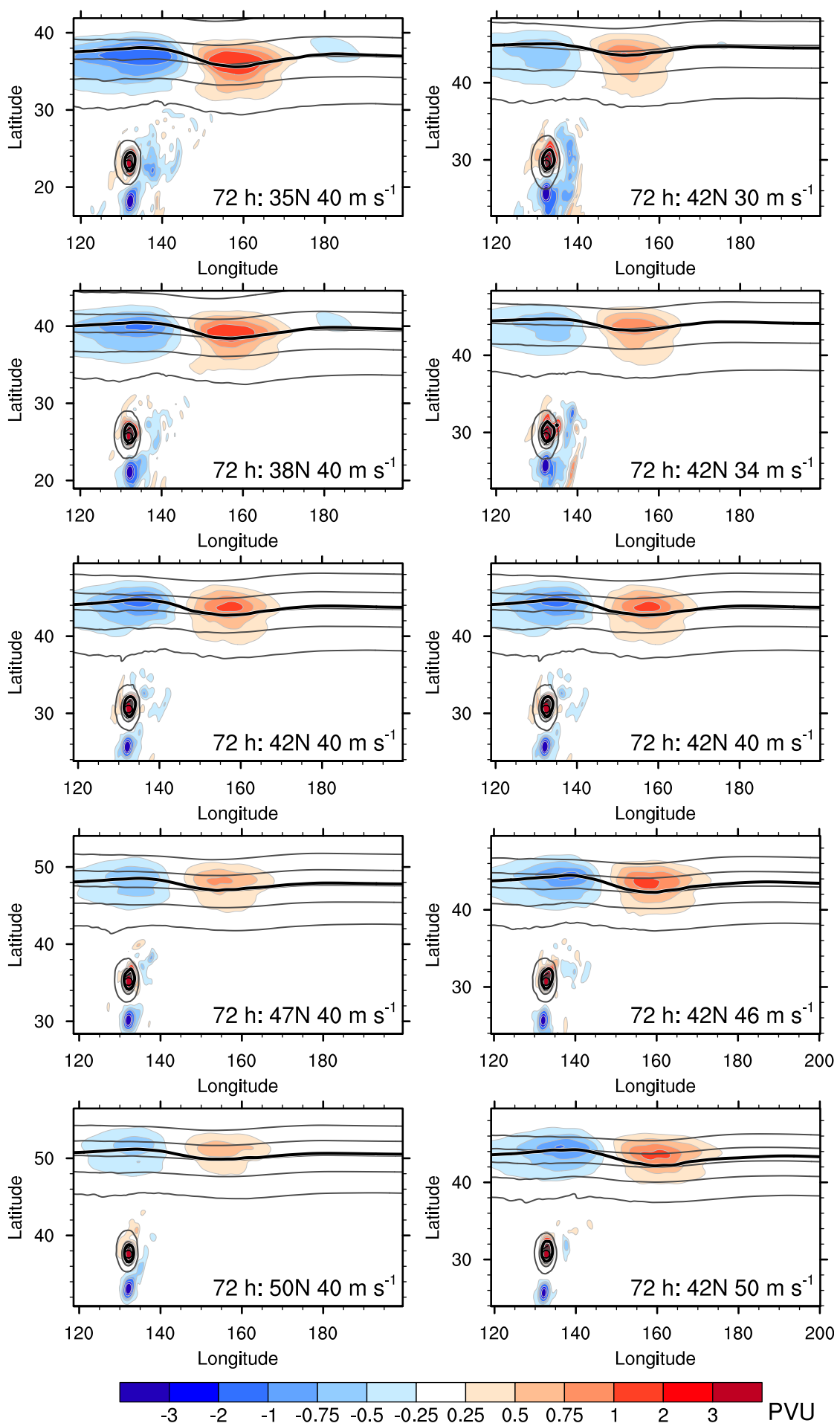

FIG. 7. Scaled anomalies in $345-\mathrm{K}$ isentropic potential vorticity relative to $t=0 \mathrm{~h}$ (shading, see text for details on scaling), 2-PVU contour of 345-K isentropic PV (thick black line), and $500-\mathrm{hPa}$ geopotential height (thin black lines from 5700 to $5900 \mathrm{~m}$ every $50 \mathrm{~m}$ ) at $t=72 \mathrm{~h}$ for (left) five simulations with $40 \mathrm{~m} \mathrm{~s}^{-1}$ jets and (right) five simulations with jets initially at $42.5^{\circ} \mathrm{N}$. 

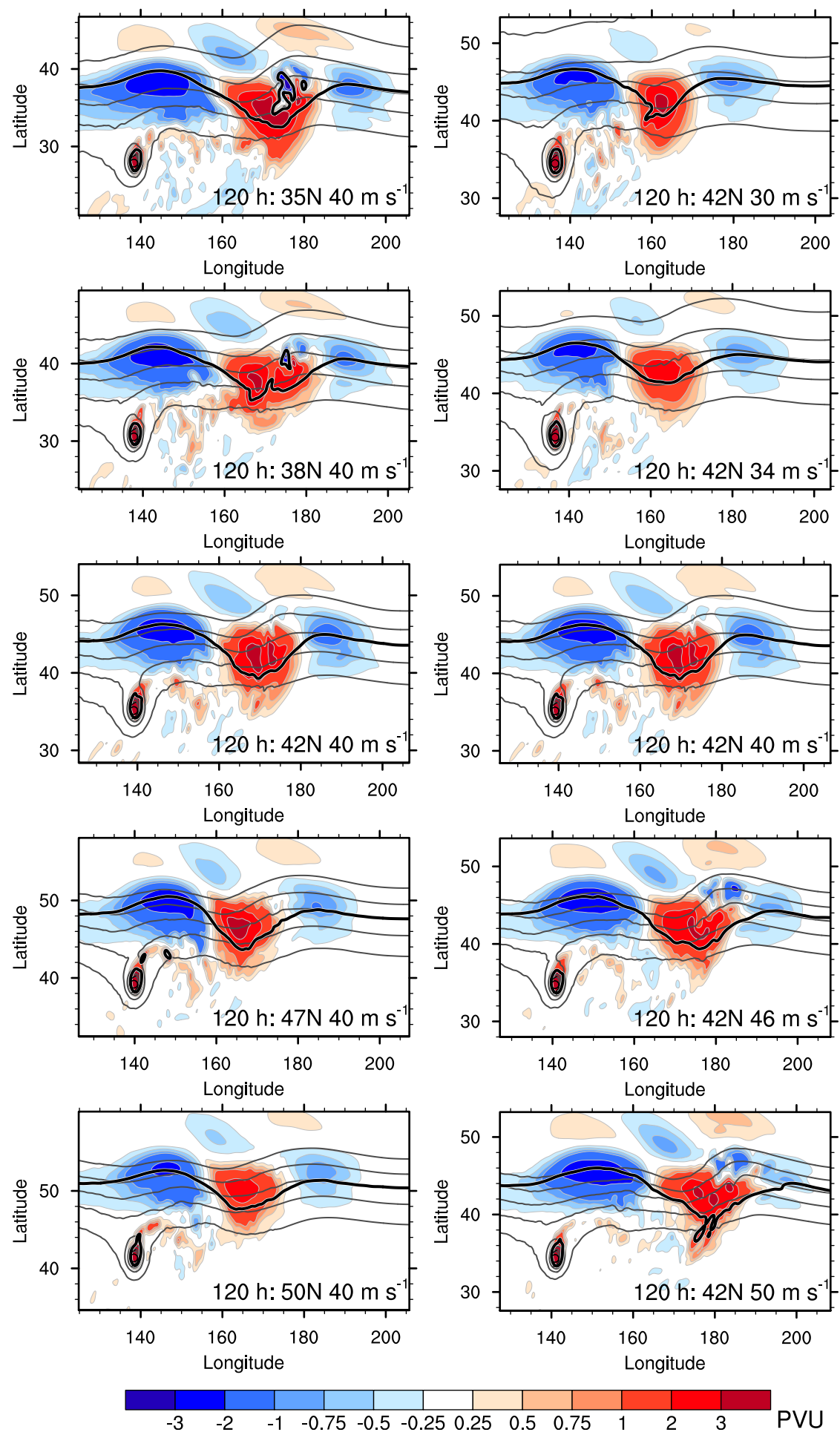

FIG. 8. As in Fig. 7, but at $t=120 \mathrm{~h}$.

downstream is the focus of the following section. But before discussing the downstream response, we briefly examine how the speed and latitude of the jets affect the intensity evolution of the TCs.
For jets initialized at the same latitude, the separation of shear traces in Fig. $3 \mathrm{c}$ according to jet speed, which begins around $60 \mathrm{~h}$ and persists through most of the simulations, is intuitive in that the TCs approaching 
(a) $\mathrm{t}=84 \mathrm{~h}$ pmin $=950.1 \mathrm{hPa}$ shear $=21.3 \mathrm{~m} \mathrm{~s}$
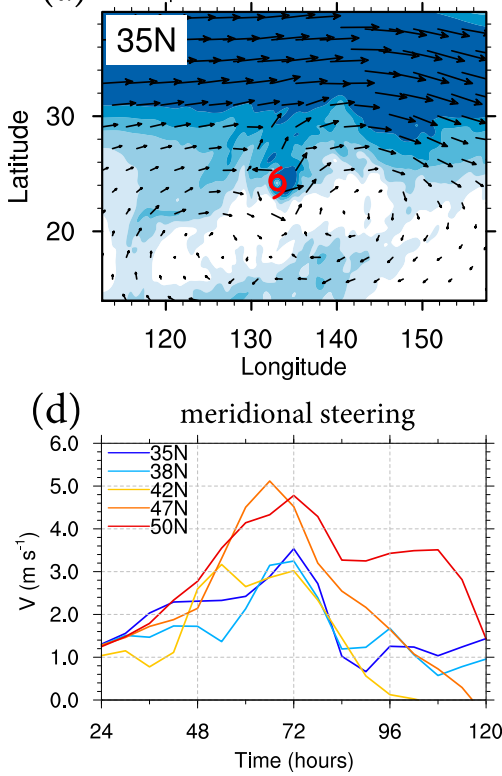

(b)

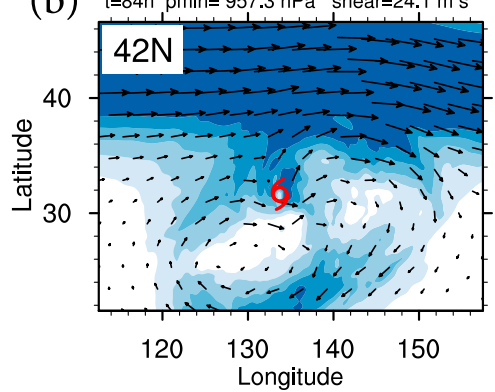

(e)

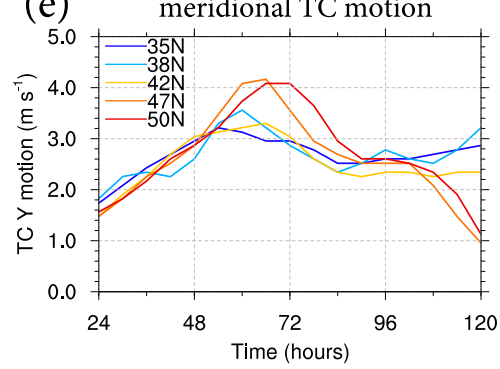

(c) $\mathrm{t}=84 \mathrm{~h}, \mathrm{pmin}=958.4 \mathrm{hPa}$ shear $=29.0 \mathrm{~m} \mathrm{~s}^{-1}$

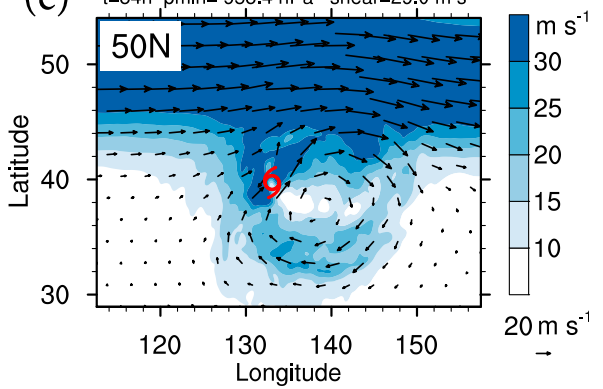

(f)

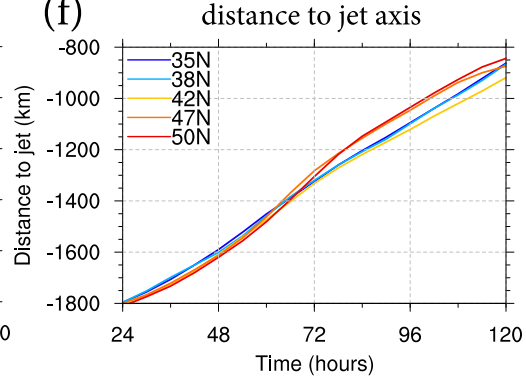

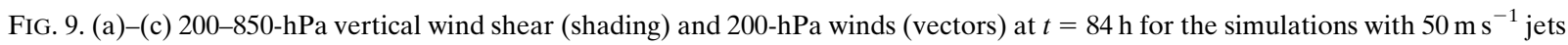
initially at (a) $35^{\circ}$, (b) $42.5^{\circ}$, and (c) $50^{\circ} \mathrm{N}$. Red TC symbols denote the position of the TC, and values printed along the top of the panels are minimum sea level pressure of the TC and the 200-850-hPa vertical wind shear averaged in the northern semicircle of the TC $100-500 \mathrm{~km}$ from the TC center. (d)-(f) Time series for the five simulations with $50 \mathrm{~m} \mathrm{~s}^{-1}$ jets of (d) the meridional winds averaged horizontally within $200 \mathrm{~km}$ of the TC center and vertically from 5 to $12 \mathrm{~km}$, (e) the meridional component of TC motion, and (f) the distance between the TC and the initial jet axis.

stronger jets (thicker lines) experience stronger vertical wind shear. This is especially evident for the jets initialized at $47^{\circ}$ and $50^{\circ} \mathrm{N}$. By $84 \mathrm{~h}$, the deep-layer vertical wind shear magnitude begins to explain more than $40 \%$ of the variance in 24-h TC intensity change over all 25 simulations, as the TCs in stronger shear begin to weaken (Fig. 3b). Figure 3c also shows periods in which the vertical wind shear exhibits a dependency on jet latitude that is less intuitive. Over the first $72 \mathrm{~h}$ of the simulations, the TCs in the low-latitude jet simulations tend to experience stronger shear than the TCs in the high-latitude jet simulations. That this is also the case for TCs in environments without a jet suggests that these shear differences relate to differences in the beta gyres at these times. Specifically, we speculate that the stronger meridional gradient in planetary vorticity toward the equator may result in stronger planetary vorticity advection by the lower-latitude TCs that enhances the beta gyres and the shear that they induce. The stratification of shear by jet latitude reverses from 84 to $120 \mathrm{~h}$, when the TCs in the higher-latitude jet simulations tend to experience stronger shear (Fig. 3c). This is especially apparent among the $50 \mathrm{~m} \mathrm{~s}^{-1}$ jets, for which the shear at $120 \mathrm{~h}$ is $5 \mathrm{~m} \mathrm{~s}^{-1}$ stronger in the $50^{\circ} \mathrm{N}$ jet simulation than in the $35^{\circ} \mathrm{N}$ jet simulation. TCs in the higher-latitude jet simulations also exhibit some of the hallmark responses to increasing vertical wind shear, such as weakening (Fig. 3b) and an increase in vortex tilt (not shown).

There is evidence from our simulations that the way in which the TC outflow anticyclone changes at higher latitudes might explain why high-latitude TCs experience stronger vertical wind shear from 84 to $120 \mathrm{~h}$. Figures $9 \mathrm{a}-\mathrm{c}$ depict snapshots at $84 \mathrm{~h}$ of the $200-\mathrm{hPa}$ wind vectors and the $200-850 \mathrm{hPa}$ vertical wind shear magnitude (shading) for the simulations with $50 \mathrm{~m} \mathrm{~s}^{-1}$ jets at $35^{\circ}, 42.5^{\circ}$, and $50^{\circ} \mathrm{N}$. The radius of the outflow anticyclone decreases as the initial jet latitude increases due to the inverse proportionality between the Rossby radius of deformation and the Coriolis parameter $f$. But how does this affect the vertical wind shear that the TC experiences? Wu and Emanuel (1993, 1994) demonstrated that, for sheared TCs, the advective tendency from the outflow anticyclone that is displaced slightly downshear causes the surface vortex to move to the left of the vertical wind shear vector. Figures $9 \mathrm{a}-\mathrm{c}$ suggest that the more compact outflow anticyclone at high latitudes promotes left-of-shear motion toward the jet axis by placing stronger southwesterly flow atop the surface vortex. Indeed, Fig. 9d shows that the southerly steering flow averaged from 5 to $12 \mathrm{~km}$ is $1-2 \mathrm{~m} \mathrm{~s}^{-1}$ stronger from 48 to $80 \mathrm{~h}$ for the jets at $47^{\circ}$ and $50^{\circ} \mathrm{N}\left(4-5 \mathrm{~m} \mathrm{~s}^{-1}\right)$ than for 

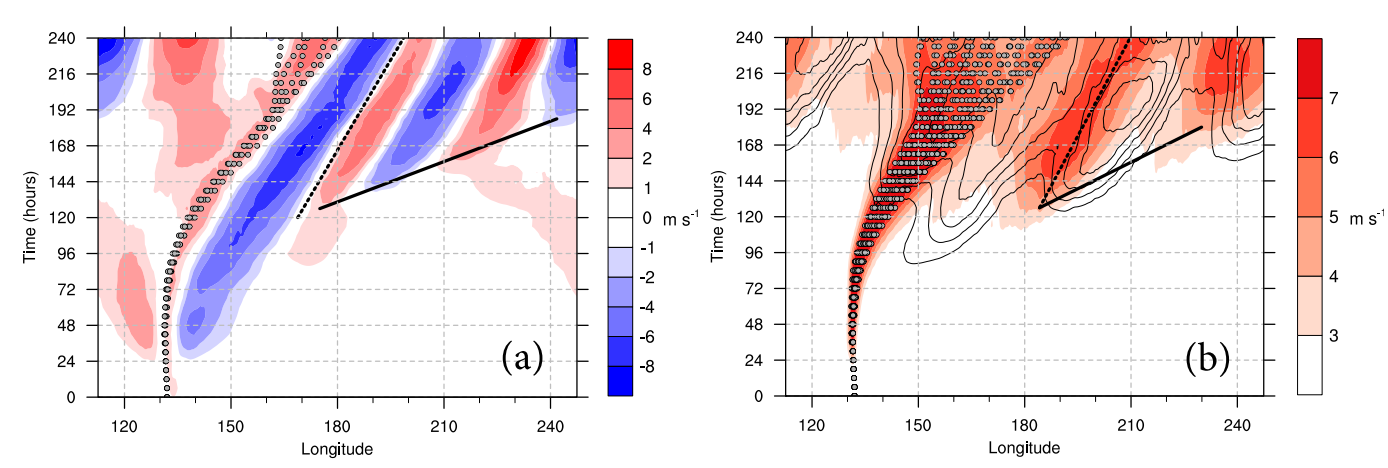

FIG. 10. Hovmöller diagram of (a) 200-hPa meridional winds averaged over the five simulations with $40 \mathrm{~m} \mathrm{~s}^{-1}$ jets, and (b) standard deviation in surface wind speed (shading) and 200-hPa meridional winds (black contours from 10 to $18 \mathrm{~m} \mathrm{~s}^{-1}$ every $2 \mathrm{~m} \mathrm{~s}^{-1}$ ) over all 25 simulations. Fields are meridionally averaged in a band that extends $1000 \mathrm{~km}$ north and south of the initial jet axis. Gray dots in (a) denote 6-hourly TC longitudes. The solid black line in each panel tracks the leading edge of a Rossby wave packet and the dashed black line denotes the primary downstream trough axis.

the lower-latitude jets $\left(2-3 \mathrm{~m} \mathrm{~s}^{-1}\right)$. During the same time period, the northward component of TC motion is up to $1 \mathrm{~m} \mathrm{~s}^{-1}$ faster for the high-latitude jets (Fig. 9e). As a result, the higher-latitude TCs end up about $100 \mathrm{~km}$ closer to the jet axis by $84 \mathrm{~h}$ (Fig. 9f). Given the strong meridional gradients in vertical wind shear near the TC at this time, even this small difference in position results in a large difference in the vertical wind shear in the northern quadrants of the TC (printed atop Figs. 9a-c). The more compact outflow anticyclone at higher latitudes also appears to enhance the vertical wind shear in a southwest to northeast-oriented axis crossing directly over the TC (Figs. 9b,c). This feature is also apparent in the $46 \mathrm{~m} \mathrm{~s}^{-1}$ jet simulations (not shown) and suggests a potentially self-destructive characteristic of high-latitude recurving TCs.

Up to this point, we have demonstrated that Rossby wave packet initiation is more sensitive to the initial latitude than the speed of the jet, with the lowestlatitude jets being the most conducive for the formation of a jet streak and a ridge immediately downstream of the TCs. We have also found stronger and higherlatitude jets to be more destructive to the TC. The remainder of this paper focuses on the implications of these findings for how the flow farther downstream of the TC evolves during the second half of the simulations (120-240 h).

\section{b. Downstream flow: Evolution and sensitivity to the initial jet}

The flow amplification that is often observed far downstream of recurving TCs is widely understood to be a result of downstream development (Simmons and Hoskins 1979; Orlanski and Sheldon 1995; Riemer et al.
2008). Because the group velocity of a Rossby wave packet exceeds the phase propagation of individual troughs and ridges within the packet, the leading edge of the packet outpaces the eastward progression of the individual waves. This enables the development of new disturbances-primarily through top-down "type B" cyclogenesis-far downstream of recurving TCs (see recent review by Wirth et al. 2018). Downstream development is seen most clearly in our simulations using a Hovmöller diagram of the $v$ winds at $200 \mathrm{hPa}$ averaged over the five simulations with $40 \mathrm{~m} \mathrm{~s}^{-1}$ jets (Fig. 10a). The TCs (gray circles) remain upstream of an amplifying ridge throughout most of the simulations, with additional troughs and ridges forming farther downstream after $120 \mathrm{~h}$. The eastward phase velocity of the primary downstream trough, estimated from the slope of the trough axis (dashed black line in Fig. 10a), is approximately $8 \mathrm{~m} \mathrm{~s}^{-1}$. The slope of the solid line, which tracks the leading edge of the Rossby wave packet, corresponds to a group velocity of approximately $19 \mathrm{~m} \mathrm{~s}^{-1}$, or $48 \%$ of the initial maximum westerly wind speed of the jet. As a fraction of the maximum jet velocity, this is considerably slower than what Simmons and Hoskins (1979) found in their dry simulations of baroclinic cyclones but in better agreement with what Riemer et al. (2008) found for TCs. Riemer et al. (2008) speculated that the slower downstream development in their simulations is due to the slower eastward motion of TCs compared to baroclinic cyclones and the upper-level TC outflow impeding the downstream propagation of wave energy.

We can begin to get a sense for how jet speed or latitude affect the amplification of waves along the jet by comparing 500-hPa geopotential height contours among subsets of the simulations. Figure 11 depicts the 5800-m 
(a)
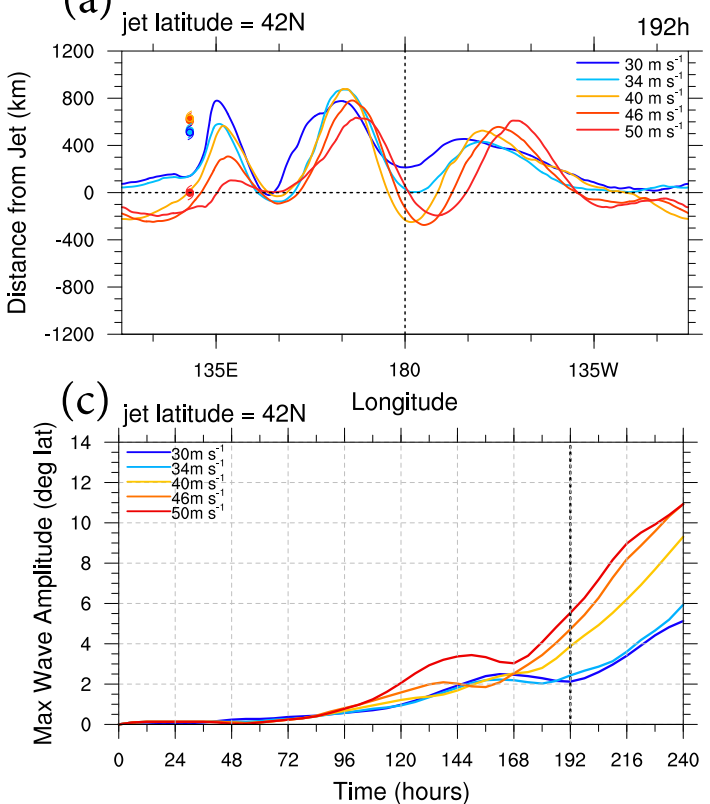

(b)

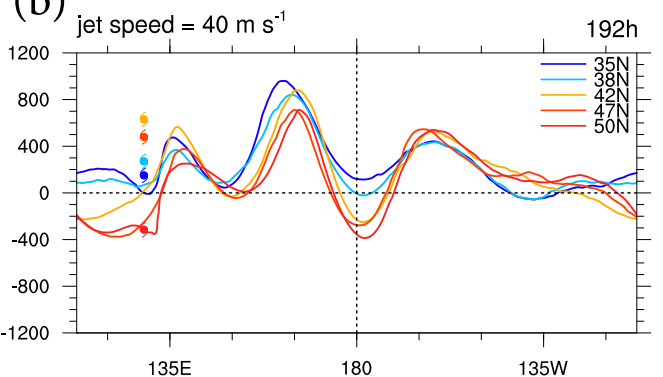

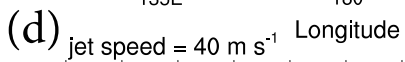

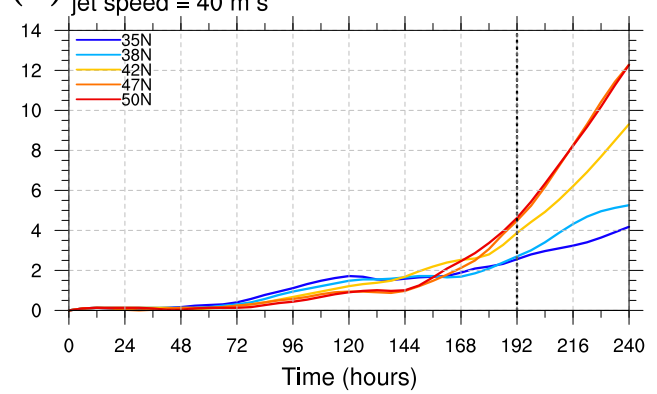

FIG. 11. The 5800-m contours of 500-hPa geopotential height at $t=192 \mathrm{~h}$ for (a) five simulations with jets initially at $42.5^{\circ} \mathrm{N}$ and (b) five simulations with $40 \mathrm{~m} \mathrm{~s}^{-1}$ initial jets. Data are shifted to be TC-relative in longitude and jet axis-relative in latitude. TC symbols indicate the location of the TC relative to the initial jet axis after zonally shifting the data. (c),(d) Time series of the maximum meridional distance of the 5800-m contour from its initial position in the region east of $180^{\circ}$ longitude (after zonally shifting the data) for the simulations corresponding to (a) and (b), respectively. The vertical dashed black line represents the time depicted in (a) and (b) (192 h).

height contour of 500-hPa geopotential height ${ }^{3}$ for five simulations with jets at $42.5^{\circ} \mathrm{N}$ (Fig. 11a) and five simulations with $40 \mathrm{~m} \mathrm{~s}^{-1}$ jets (Fig. 11b). The depicted time of $192 \mathrm{~h}$ is after the downstream flow has had sufficient time to amplify. To facilitate the comparison between the simulations, the geopotential height fields are shifted to be jet-relative in latitude and TC-relative in longitude. At $192 \mathrm{~h}$, the amplitude (measured as the distance of the contours from their initial latitude) of the primary downstream ridge located near $135^{\circ} \mathrm{E}$ and the trough just upstream of the TCs is larger for weaker and higherlatitude jets. Furthermore, the amplitude of the primary downstream ridge appears to depend more on jet speed than jet latitude. The more amplified primary downstream ridge in the weaker jet simulations is consistent with the sensitivity tests of Riemer et al. (2008), and may be attributed to TCs remaining stronger (Fig. 3b) and translating farther poleward in these simulations (Fig. 11a). By contrast, the second downstream trough and third downstream ridge are more amplified for stronger and higher-latitude jets at $192 \mathrm{~h}$. Time series of

\footnotetext{
${ }^{3}$ We have also examined 5700- and 5900-m contours of 500-hPa geopotential height and the results are consistent with the $5800-\mathrm{m}$ contour analysis.
}

the maximum amplitude of the 500-hPa height contours east of the vertical dashed black line in Figs. 11a and 11b confirm this; $500-\mathrm{hPa}$ wave amplitudes at $192 \mathrm{~h}$ in this far downstream region are up to twice as large on the strong or high-latitude jets as they are on weak or lowlatitude jets (Figs. 11c,d). This analysis suggests that Rossby wave packets amplify more rapidly along the stronger and higher-latitude jets as they propagate far downstream of the TCs, regardless of which jets were initially perturbed most strongly or became more amplified locally around the recurving TC. Consequently, these stronger and higher-latitude jets end up having the most amplified downstream response to recurving TCs.

That strong and high-latitude jets are more baroclinically unstable largely explains why Rossby wave packets amplify more rapidly along such jets. The Eady baroclinic growth rate estimates the growth rate of the most baroclinically unstable wavenumber. For the jets in this study, we compute the Eady growth rate at the initial time for a grid point directly underneath the jet axis following Lindzen and Farrell (1980),

$$
\sigma_{E}=0.3125 \times \frac{f}{\bar{N}}\left(\frac{u_{\mathrm{max}}-u_{\mathrm{sfc}}}{z_{\mathrm{jet}}}\right),
$$


(a) Maximum Eady growth rate $(t=0 \mathrm{~h})$

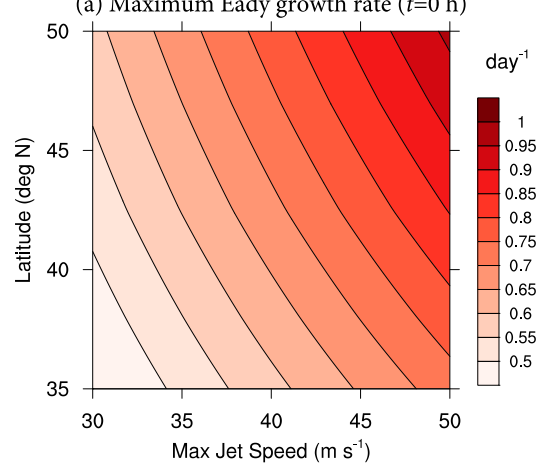

(b) Maximum intensification rate $(t=204 \mathrm{~h})$

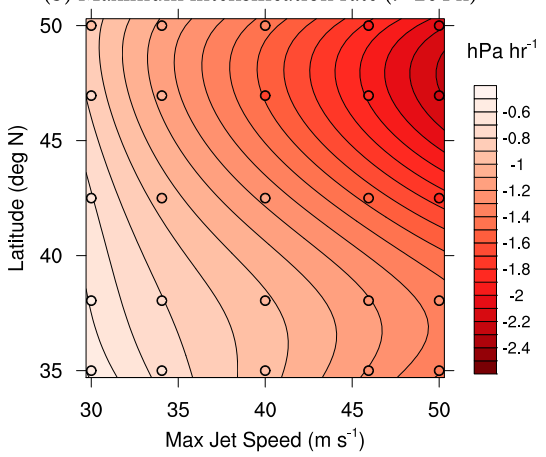

(c) $v$-wind on 1.5-PVU surface $(t=204 \mathrm{~h})$

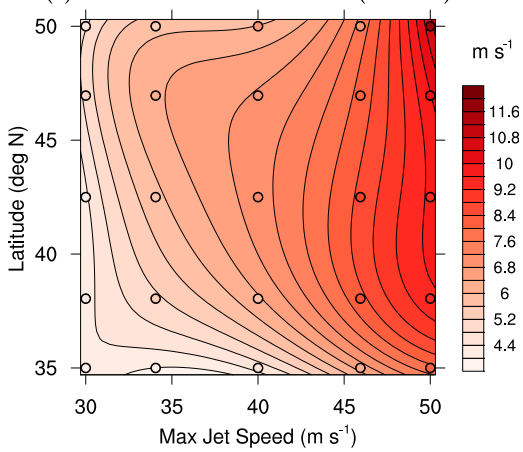

FIG. 12. (a) Eady baroclinic growth rates computed for a point directly underneath the jet axis at $t=0 \mathrm{~h}$ for each of the 25 simulations plotted in the jet speed-jet latitude input space; (b) response surface of the maximum intensification rate $\left(\mathrm{hPa} \mathrm{h}^{-1}\right)$ of downstream surface cyclones normalized by jet latitude (see text for details) at $t=204 \mathrm{~h}$, plotted in the same space as the Eady growth rate in (a); and (c) response surface of meridional winds on the 1.5-PVU surface at $t=204 \mathrm{~h}$ averaged within the same downstream region in which the maximum intensification rate in (b) is computed. Filled circles in (b) and (c) denote the jet latitudes and speeds used to initialize the 25 simulations from which the response surfaces are constructed. Circles are colored according to the maximum intensification rate or mean meridional winds computed directly from the model output at $t=204 \mathrm{~h}$.

where $f$ is the Coriolis parameter at the latitude of the jet axis, $\bar{N}$ is the mass-weighted vertical average of the Brunt-Väisälä frequency between the surface and the jet maximum level, and the term in parentheses is the bulk vertical wind shear magnitude between the surface and the jet maximum level. Figure 12a depicts $\sigma_{E}$ in a two-dimensional space spanned by the initial latitudes and maximum wind speeds of the jets. The largest Eady growth rates correspond to faster jets (due to stronger vertical wind shear) and higher-latitude jets (due to a larger Coriolis parameter). Such jets occupy the upper-right corner of Fig. 12a. Figure 12b depicts the maximum intensification rate of downstream cyclones, computed as the largest hourly decrease in surface pressure (averaged in the 12-h interval centered at 204h) within a region downstream of the TC. The downstream region extends from 1800 to $13200 \mathrm{~km}$ east of the TC and $1500 \mathrm{~km}$ north and south of the initial jet axis. Following Binder et al. (2016), we multiply the maximum intensification rate by $\sin \left(60^{\circ}\right) / \sin (\phi)$, where $\phi$ is the latitude where the largest decrease in surface pressure occurs. Values are plotted in the same two-dimensional space shown in Fig. 12a. The time of $204 \mathrm{~h}$ is chosen because it is when the largest downstream cyclone intensification rates occur. Figure $12 \mathrm{c}$ depicts the meridional wind on the 1.5-PVU surface at $204 \mathrm{~h}$ averaged in the same downstream region used for computing the intensification rate. This quantity is similar to the North Pacific meridional flow index of Archambault et al. (2013). Figures 12b and $12 \mathrm{c}$ are created by fitting a two-dimensional, fourth-order Legendre polynomial series to the respective quantities computed from the output of each simulation using the method described in Finocchio et al. (2016, their section 4). The circles in Figs. $12 \mathrm{~b}$ and $12 \mathrm{c}$ are colored according to the values of intensification rate or meridional winds computed from the model output. Differences between the model output and the response surface are generally less than $10 \%$ of the range of values on the response surface.

Qualitatively, the structure of the response surface for the maximum intensification rate of downstream cyclones (Fig. 12b) resembles the basic structure of the baroclinic growth rates (Fig. 12a). The largest intensification rates occur along the most baroclinically unstable jets, as indicated by the darker red shading in the upperright corners of each panel. Closer inspection of Fig. 12b also reveals that the gradients along the response surface are stronger for the more baroclinically unstable jets. For example, the difference in downstream cyclone intensification rates between the strongest and the weakest jets increases from $0.9 \mathrm{hPah}^{-1}$ at $35^{\circ} \mathrm{N}$ to $1.2 \mathrm{hPa} \mathrm{h}^{-1}$ at $50^{\circ} \mathrm{N}$. Differences in the downstream cyclone intensification rates between the highest- and lowestlatitude jet simulations increase by a similar amount going from the 30 to the $50 \mathrm{~m} \mathrm{~s}^{-1}$ jets. This suggests downstream cyclone intensification becomes more sensitive to changing the speed or latitude of the jet as the jet becomes more baroclinically unstable.

Unlike surface pressure, the upper-level wind response downstream of the TCs (Fig. 12c) is primarily a function of jet speed - especially for faster jets initialized poleward of $40^{\circ} \mathrm{N}$. Partitioning the variance between jet speed and jet latitude based on the coefficients of the polynomial series expansion used to create the response surfaces indicates that jet speed explains $84 \%$ of the variance in the upper-level meridional winds and $66 \%$ of the variance in the intensification rate at $204 \mathrm{~h}$. Some of the enhanced sensitivity of the upper-level winds to the 

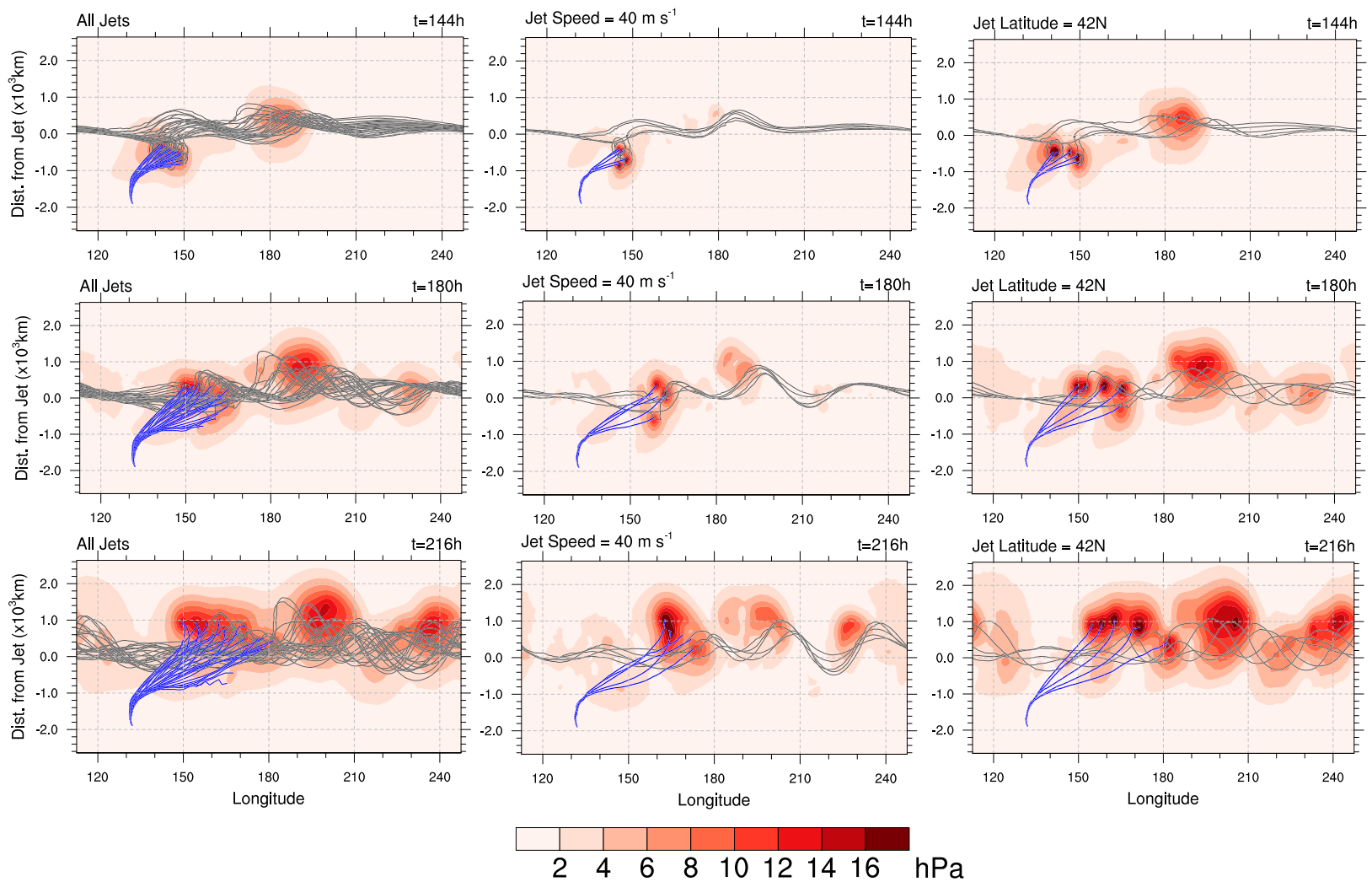

FIG. 13. Standard deviation of surface pressure (shading), 5800-m contours of 500-hPa geopotential height (gray), and TC tracks (blue) at (top) $t=144$, (middle) $t=180$, and (bottom) $t=216 \mathrm{~h}$ for (left) all 25 simulations, (center) the five simulations with $40 \mathrm{~m} \mathrm{~s}^{-1}$ jets, and (right) the five simulations with the initial jet axis at $42.5^{\circ} \mathrm{N}$. Data are shifted to be jet-relative in latitude.

initial speed of the jet could be due to the weakening of the geostrophic winds associated with a given pressure gradient as the Coriolis parameter increases with latitude.

To better understand the spatial characteristics of the downstream response and its sensitivity to the initial jet, we also analyze geographic distributions of differences among the simulations. Figure 13 depicts snapshots of the standard deviation (hereafter "spread") in surface pressure computed in a jet-relative framework for all 25 simulations (left column) and for subsets of the simulations (right columns). In the full set of simulations, an area of elevated spread is evident at $144 \mathrm{~h}$ near $180^{\circ}$ longitude, about $3000 \mathrm{~km}$ downstream of the TCs (topleft panel of Fig. 13). The spread within this region amplifies over the next 3 days to $15 \mathrm{hPa}$, as an additional center of elevated spread develops about $5000 \mathrm{~km}$ downstream of the TCs, between $230^{\circ}$ and $240^{\circ}$ longitude (middle- and bottom-left panels of Fig. 13). These areas of elevated spread reflect variability in the position and intensity of developing surface cyclones and anticyclones. Moreover, Fig. 10b shows that the spread in surface and upper-level winds computed over all
25 simulations has similar downstream propagation characteristics to the Rossby wave packets diagnosed from Fig. 10a. This indicates that the spread among the simulations propagates downstream with the wave packets triggered during the TC-jet interaction (Aiyyer 2015; Torn 2017).

The right two columns of Fig. 13 depict the same fields as the left column, but for subsets of five simulations in which only the initial jet latitude differs (middle column) or the initial jet speed differs (right column). Jet latitude differences produce the smallest spread in surface pressure and only slight differences in the amplitude of waves at $500 \mathrm{hPa}$ during the depicted times. Jet speed differences, however, result in spread that is comparable in magnitude to the spread over all 25 simulations by $216 \mathrm{~h}$, as well as large differences in the amplitude and phase of 500-hPa waves.

It is unclear from Fig. 13 how much of the phase variability among the different jet speed simulations comes from the zonal spread in TC tracks, which triggers Rossby wave packets at different longitudes along the jet, versus how much comes from differences in the propagation of Rossby waves away from their point of 

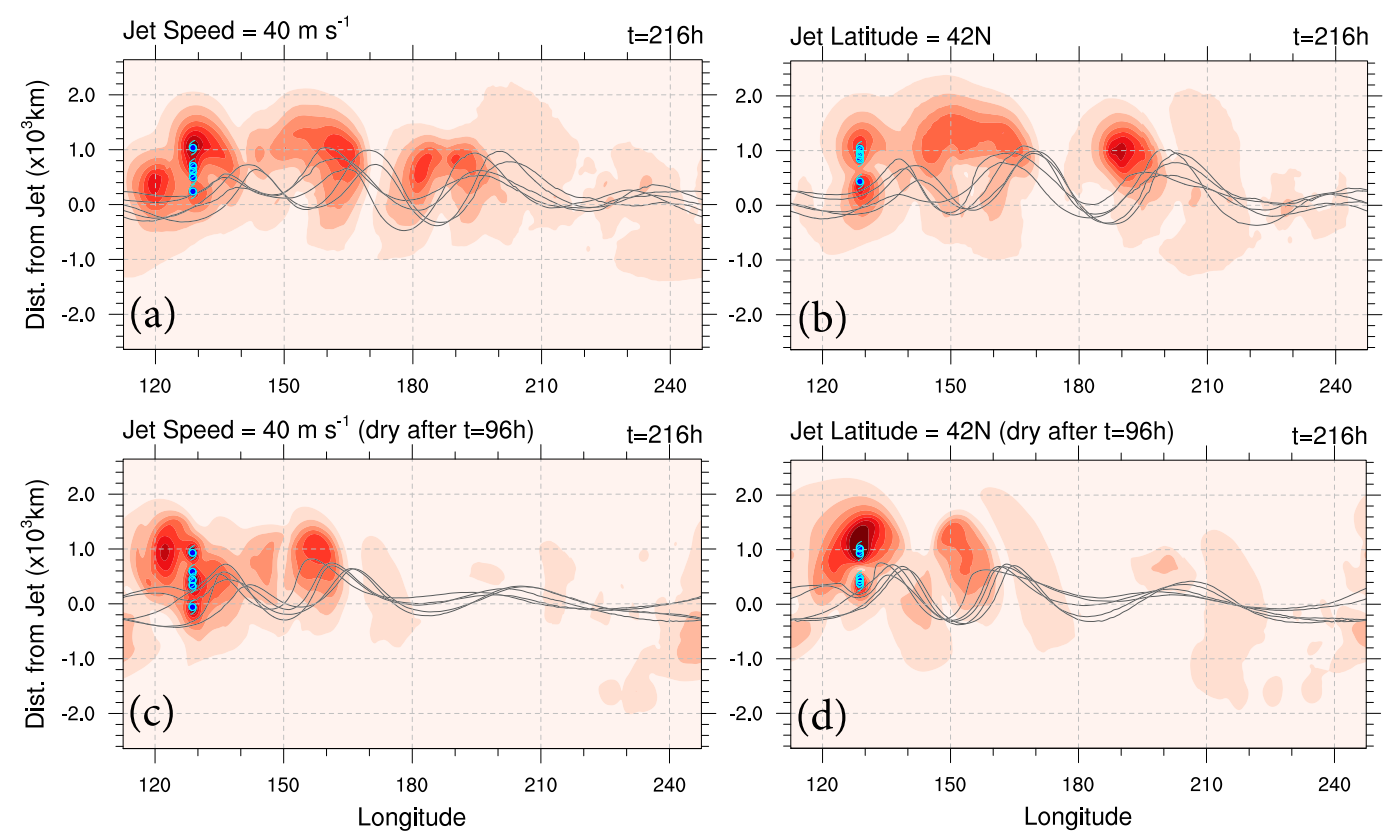

$\begin{array}{llllllllll}2 & 4 & 6 & 8 & 10 & 12 & 14 & 16 & \mathrm{hPa}\end{array}$

FIG. 14. Standard deviation of surface pressure (shading) and 5800-m contours of 500-hPa geopotential height (gray) for (a) five full-physics simulations with $40 \mathrm{~m} \mathrm{~s}^{-1}$ jets, (b) five full-physics simulations with jets initially at $42.5^{\circ} \mathrm{N}$, (c) five simulations without condensational heating after $96 \mathrm{~h}$ (see text for details) and with $40 \mathrm{~m} \mathrm{~s}^{-1}$ jets, and (d) five simulations without condensational heating after $96 \mathrm{~h}$ and with jets initially at $42.5^{\circ} \mathrm{N}$. Data are shifted to be jet-relative in latitude and TC-relative in longitude before computing standard deviations. Cyan TC symbols indicate the location of the TC relative to the initial jet axis after zonally shifting the data.

origin. To address this ambiguity, we recompute the spread at $216 \mathrm{~h}$ for each set of five simulations shown in the right columns of Fig. 13 after shifting the data to be both jet-relative in latitude and TC-relative in longitude (Figs. 14a,b). The pattern of downstream spread at $216 \mathrm{~h}$ for the five simulations in which only the latitude of the jet differs still exhibits two centers of spread exceeding $8 \mathrm{hPa}$ located 3000 and $5000 \mathrm{~km}$ downstream of the recurving TCs, at $155^{\circ}$ and $190^{\circ}$ longitude (Fig. 14a). Shifting the data from the variable jet speed simulations (Fig. 14b) reduces the phase differences between the Rossby waves in each simulation, focusing the downstream spread in smaller regions around the downstream surface cyclones. Nevertheless, the spread in the vicinity of the second downstream cyclone remains around $15 \mathrm{hPa}-$ comparable to Fig. 13 . So while the differences in TC tracks are indeed a source of spread in some areas downstream of the recurving TCs in Fig. 13, after accounting for TC track differences, changing the speed of the jet still results in larger spread in surface pressure around the cyclones furthest downstream of the TC than changing the initial latitude of the jet. This result corroborates that the downstream response to recurving TCs in our simulations is more sensitive to differences in the speed rather than the latitude of the jet.

\section{Role of moist diabatic processes}

This section describes an additional set of simulations in which we disable moist physical processes after the TCs intensify and interact with the jet. The purpose of these simulations is to isolate the role of condensational heating in the amplification of the downstream response and the growth of downstream spread among the simulations. Each additional simulation is restarted at $96 \mathrm{~h}$, when the TCs are near their maximum intensities (Fig. 3b) and when a jet streak and downstream ridge have been established. The cumulus and microphysics schemes are bypassed after restarting the model, eliminating condensation as a diabatic heat source. To prevent TCs from spinning down in the absence of condensational heating, the surface stress is also set to zero after restarting the model. Due to the lack of surface stress, all of the TCs in the dry simulations intensify between 180 and $240 \mathrm{~h}$-some reaching lifetime maximum intensities of $933 \mathrm{hPa}$ - while the TCs in the fully moist simulations weaken on average during this 
(a)
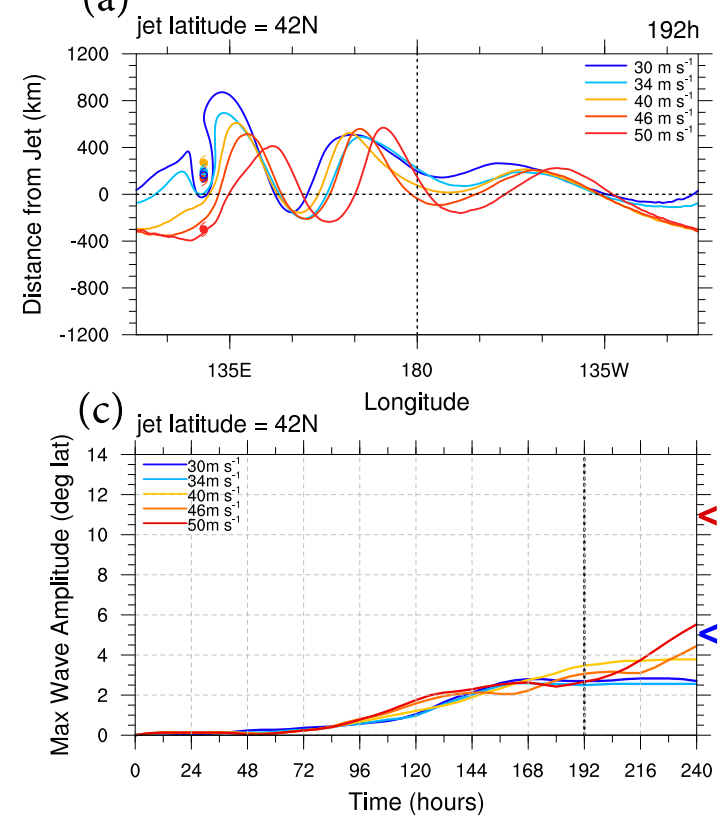

(b)

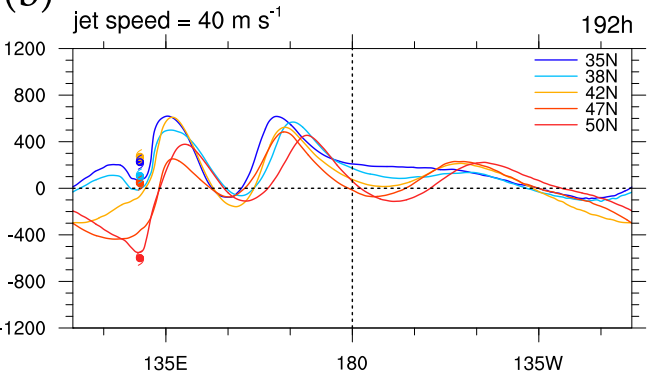

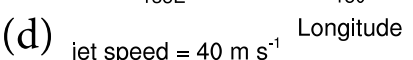

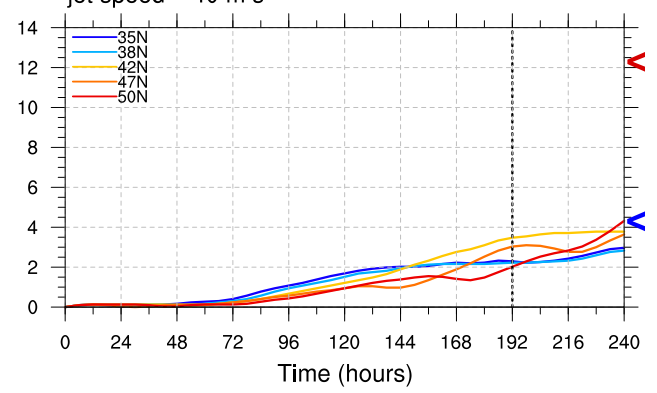

FIG. 15. As in Fig. 11, but for simulations with condensational heating deactivated after $96 \mathrm{~h}$ (see text for details). For ease of comparison with Figs. 11c and 11d, the left arrows along the right $y$ axis indicate wave amplitudes at $240 \mathrm{~h}$ from the fully moist simulations for the 30 (blue) and $50 \mathrm{~m} \mathrm{~s}^{-1}$ (red) jets in (c), and for the $35^{\circ}$ (blue) and $50^{\circ} \mathrm{N}$ (red) jets in (d).

interval. Our concern in this section, however, is not on the evolution of the transitioning TCs but on the evolution of the downstream flow. ${ }^{4}$ Presumably, a lack of friction would also promote stronger downstream cyclones, but we find this not to be the case; the minimum downstream surface pressures are higher on average in the dry simulations. As will soon be evident, the weaker downstream surface cyclones in the dry simulations likely reflect weaker top-down forcing for downstream cyclogenesis as a result of less-amplified upper-level Rossby waves.

Similar to Fig. 11, Fig. 15 illustrates the 5800-m contour of 500-hPa geopotential height at $192 \mathrm{~h}$ and time series of the maximum downstream wave amplitude at $500 \mathrm{hPa}$ for five dry simulations with jets initially at $42.5^{\circ} \mathrm{N}$ (Figs. $15 \mathrm{a}, \mathrm{c}$ ) or with $40 \mathrm{~m} \mathrm{~s}^{-1}$ jets (Figs. 15b,d). Comparing Figs. 11a,b and 15a,b, the primary downstream ridge is actually more amplified at $192 \mathrm{~h}$ in the dry simulations. The more intense TCs/extratropical cyclones that result from turning off surface stress after

\footnotetext{
${ }^{4}$ We conducted an additional simulation in which the surface stress is left unchanged after deactivating moist physics. As expected, the TC rapidly weakens between 96 and $120 \mathrm{~h}$, but the evolution of the downstream flow between 120 and $240 \mathrm{~h}$ is remarkably similar to that of the dry simulation without surface stress, despite the significantly weaker TC.
}

$96 \mathrm{~h}$ in the dry simulations might explain the more amplified primary downstream ridge, as the stronger cyclonic circulation near the surface would enhance the poleward advection of warm, moist air into the primary downstream ridge (Riemer et al. 2008; Riemer and Jones 2010). Despite the higher-amplitude ridge immediately downstream of the TC, the farthest downstream ridge between $165^{\circ}$ and $135^{\circ} \mathrm{W}$ is considerably less amplified in the dry simulations at $192 \mathrm{~h}$ (Figs. 15a,b) than in the moist simulations at the same time (Figs. 11a,b). By $240 \mathrm{~h}$, the maximum amplitude of the depicted contour in the eastern half of the domain is between 1.5 and 3 times higher in the moist simulations than in the dry simulations (Figs. 15c,d). Deactivating condensational heating also appears to render the downstream wave amplitude after $120 \mathrm{~h}$ less sensitive to both the initial speed and latitude of the jet.

The reduced downstream sensitivity to changing the speed or latitude of the jet in the dry simulations is also evident near the surface. Figures $14 \mathrm{c}$ and $14 \mathrm{~d}$ depict the spread in surface pressure due to jet latitude (Fig. 14c) and jet speed (Fig. 14d) in the dry simulations at $216 \mathrm{~h}$. As in the fully moist simulations (Figs. 14a,b), there is a distinct center of elevated spread located near $155^{\circ}$ longitude or about $3000 \mathrm{~km}$ downstream of the TCs. The magnitude of spread within this region is comparable among the dry and moist simulations. The largest 
differences in spread between the dry and moist simulations appear farther downstream, where a second area of elevated spread in excess of $8 \mathrm{hPa}$ that appears in both subsets of the moist simulations is nearly absent in the dry simulations. These findings underscore the importance of moist diabatic processes for both amplifying downstream waves and enhancing their sensitivity to the speed or latitude of the jet stream. However, a more detailed analysis is needed to determine the relative importance of diabatic heating local to the developing downstream cyclones versus that associated with their warm conveyor belts (Grams and Archambault 2016).

\section{Discussion and conclusions}

The ability of a recurving TC to excite Rossby waves and alter the weather far downstream requires a sufficient interaction between the TC and the jet stream, a zonally extensive jet stream to transmit the Rossby wave energy long distances from the TC, and sufficient baroclinic instability along the jet stream for Rossby waves to amplify. In this study, we have found that the baroclinicity along the jet is a key, and perhaps underappreciated, determinant of the extent to which recurving TCs affect the downstream flow.

In a set of 25 simulations using a nonhydrostatic mesoscale model in which the initial speed and latitude of a zonal, upper-tropospheric jet is changed over a range of realistic values, the fastest and highest latitude (i.e., the most baroclinically unstable) jets consistently produce a more amplified and variable downstream response. The apparent importance of baroclinic instability in determining the downstream response and its variability in our simulations add generality to what Reynolds et al. (2009) found for a sample of typhoons in the western North Pacific. In that study, recurving typhoons with the largest growth in singular vector perturbation energy over 5 days were characterized by higher downstream baroclinic instability. Therefore, known geographic differences in the likelihood for real TCs to induce an amplified downstream response (e.g., Torn and Hakim 2015; Torn 2017) may relate to differences in the mean structure and baroclinicity of the jet.

A surprising result of this study is how insensitive downstream Rossby wave amplification is to the TC and its interactions with the jet. Due to the stronger vertical wind shear, TCs in the simulations with faster jets tend to weaken earlier and remain weak as extratropical cyclones (Fig. 3b), yet the faster jets end up having a more amplified downstream response. This weak relationship between the intensity of the TC and the amplitude of the downstream response agrees with several previous studies (Archambault et al. 2013; Quinting and Jones
2016; Riboldi et al. 2018). However, in contrast with previous studies (Archambault et al. 2015; Quinting and Jones 2016; Grams and Archambault 2016; Keller et al. 2019), we find the strength of the interaction between the TC and the jet does not necessarily determine the amplitude of the downstream response either. For example, the reduced environmental inertial stability in the TC outflow layer at lower latitudes allows for an earlier and stronger interaction between TCs and lowlatitude jets (as measured by the formation of a jet streak and upper-level PV anomalies immediately downstream of the TC), yet the high-latitude jets have a more amplified downstream response. In their composite analysis of straight jets, Riboldi et al. (2018) found the differences in PV advection by the irrotational outflow to be fairly small between composites of cases with and without downstream Rossby wave initiation. Whether the apparent insensitivity of the downstream response to TC-jet interactions is unique to the predominately straight jets analyzed here and in Riboldi et al. (2018) warrants further investigation. In any case, our simulations highlight the important role of jet stream baroclinicity in amplifying the downstream flow following TC recurvature.

An advantage of the full-physics idealized modeling framework used in this study is the capability to explicitly examine the role of moist physics in the generation of the downstream response. A series of simulations in which moist diabatic processes in the model are deactivated after the initiation of a Rossby wave packet reveals that the amplitude of downstream waves is reduced by as much as a factor of 3 compared to the fully moist simulations. This reduction in the downstream wave amplitude is consistent with what Grams and Archambault (2016) found in a similar simulation with latent heat release turned off. We also find the spread associated with the farthest downstream cyclone in these simulations is reduced by up to a factor of 3 . Presumably, if the simulations were extended beyond 10 days, then the downstream waves (and the spread among them) would eventually amplify to have similar magnitudes as found in the fully moist simulations. Nevertheless, a key result of this study is that condensational heating reduces the time it takes for the development of a high-amplitude downstream response to between 7 and 10 days.

Previous studies have linked the observed seasonality of the downstream response to recurving TCs to seasonal variations in the jet stream (Reynolds et al. 2009; Archambault et al. 2013, 2015). Results from these studies and from the present study point to autumn as being an optimal time of year for highly amplified downstream responses to recurving TCs. This is due to a 
combination of the large number of TCs during autumn that can initiate wave packets coupled with the increasing baroclinicity along the restrengthening midlatitude jet. Lillo and Parsons (2017) also find September and October to have the highest frequency of forecast busts over Europe, which they attribute to Atlantic TCs recurving toward a zonally extensive midlatitude waveguide. While our results do not explicitly demonstrate how the initial speed or latitude of the jet stream affects downstream predictability, we do find that the downstream response becomes increasingly sensitive to changing the initial speed or latitude of the jet as the jets become more baroclinically unstable.

We have made several idealizations to the jet stream in this study that limit the extent to which our simulations can be assumed to represent reality. For example, by initializing jets without Rossby waves, we neglect phasing uncertainties between TCs and upstream troughs that are known to cause downstream forecast uncertainty (Scheck et al. 2011; Grams et al. 2013b). In addition, the zonally continuous jets in this study all but guarantee that the Rossby wave response is able to propagate far downstream of the TC, which is why virtually all of our simulations exhibit some degree of downstream flow amplification. Observed jet streams are often characterized by zonal discontinuities, which serve as focal points for Rossby wave breaking (Postel and Hitchman 1999) that could prevent far-downstream flow amplification from occurring as consistently as it does in our simulations. At the same time, Rossby wave breaking can enhance downstream forecast uncertainty by inducing difficult-to-forecast pattern changes, such as the generation or destruction of atmospheric blocks, potentially leading to large forecast busts. Systematically linking predictability and the likelihood of forecast busts following TC recurvature to the structural characteristics and baroclinicity of the jet stream is a topic worthy of further investigation, and for which this study provides a starting point.

Acknowledgments. We are grateful to three anonymous reviewers whose comments greatly improved this manuscript. We also thank David Ryglicki and Dan Hodyss at the Naval Research Laboratory for their assistance with the Helmholtz decomposition. This research was performed while the first author held an NRC Research Associateship award at the Naval Research Laboratory Marine Meteorology Division. This research is supported by the Chief of Naval Research through the NRL Base Program, PE 0601153N. Computational resources were supported in part by a grant of HPC time from the Department of Defense Major Shared Resource Centers, Stennis Space Center, MS.
COAMPS is a registered trademark of the Naval Research Laboratory.

\section{REFERENCES}

Aiyyer, A. R., 2015: Recurving tropical cyclones and mid-latitude predictability. Geophys. Res. Lett., 42, 7799-7807, https:// doi.org/10.1002/2015GL065082.

Anwender, D., P. A. Harr, and S. C. Jones, 2008: Predictability associated with the downstream impacts of the extratropical transition of tropical cyclones: Case studies. Mon. Wea. Rev., 136, 3226-3247, https://doi.org/10.1175/2008MWR2249.1.

Archambault, H. M., L. F. Bosart, D. Keyser, and J. Cordeira, 2013: A climatological analysis of the extratropical flow response to recurving western North Pacific tropical cyclones. Mon. Wea. Rev., 141, 2325-2346, https://doi.org/10.1175/MWR-D12-00257.1.

— D. Keyser, L. F. Bosart, C. A. Davis, and J. Cordeira, 2015: A composite perspective of the extratropical flow response to recurving western North Pacific tropical cyclones. Mon. Wea. Rev., 143, 1122-1141, https://doi.org/10.1175/MWR-D14-00270.1.

Binder, H., M. Boettcher, H. Joos, and H. Wernli, 2016: The role of warm conveyor belts for the intensification of extratropical cyclones in Northern Hemisphere winter. J. Atmos. Sci., 73, 3997-4020, https://doi.org/10.1175/JAS-D-15-0302.1.

Bosart, L. F., B. J. Moore, J. M. Cordeira, and H. M. Archambault, 2017: Interactions of North Pacific tropical, midlatitude, and polar disturbances resulting in linked extreme weather events over North America in October 2007. Mon. Wea. Rev., 145, 1245-1273, https://doi.org/10.1175/MWR-D-16-0230.1.

Bougeault, P., 1985: The diurnal cycle of the marine stratocumulus layer: A higher-order model study. J. Atmos. Sci., 42, 2826-2843, https:// doi.org/10.1175/1520-0469(1985)042<2826:TDCOTM>2.0.CO;2.

Branstator, G., 1983: Horizontal energy propagation in a barotropic atmosphere with meridional and zonal structure. J. Atmos. Sci., 40, 1689-1708, https://doi.org/10.1175/1520-0469(1983)040<1689: HEPIAB $>2.0 . \mathrm{CO} ; 2$.

Chen, S., and Coauthors, 2003: COAMPS version 3 model description - General theory and equations. NRL Publ. 448, Naval Research Laboratory, Marine Meteorology Division, Monterey, CA, 143 pp.

Doyle, J., and Coauthors, 2014: Tropical cyclone prediction using COAMPS-TC. Oceanography, 27, 104-115, https://doi.org/ 10.5670/oceanog.2014.72.

Dunion, J. P., 2011: Rewriting the climatology of the tropical North Atlantic and Caribbean Sea atmosphere. J. Climate, 24, 893908, https://doi.org/10.1175/2010JCLI3496.1.

Evans, C., and Coauthors, 2017: The extratropical transition of tropical cyclones. Part I: Cyclone evolution and direct impacts. Mon. Wea. Rev., 145, 4317-4344, https://doi.org/10.1175/ MWR-D-17-0027.1.

Finocchio, P. M., S. J. Majumdar, D. S. Nolan, and M. Iskandarani, 2016: Idealized tropical cyclone responses to the height and depth of environmental vertical wind shear. Mon. Wea. Rev., 144, 2155-2175, https://doi.org/10.1175/MWR-D-15-0320.1.

Fiorino, M., and R. L. Elsberry, 1989: Some aspects of vortex structure related to tropical cyclone motion. J. Atmos. Sci., 46, 975-990, https://doi.org/10.1175/1520-0469(1989)046<0975: SAOVSR $>2.0 . \mathrm{CO} ; 2$.

Grams, C. M., and S. R. Blumer, 2015: European high-impact weather caused by the downstream response to the extratropical 
transition of North Atlantic Hurricane Katia (2011). Geophys. Res. Lett., 42, 8738-8748, https://doi.org/10.1002/ 2015 GL066253.

, and H. M. Archambault, 2016: The key role of diabatic outflow in amplifying the midlatitude flow: A representative case study of weather systems surrounding western North Pacific extratropical transition. Mon. Wea. Rev., 144, 38473869, https://doi.org/10.1175/MWR-D-15-0419.1.

_ in modifying the upper-tropospheric wave guide: A North Atlantic case-study. Quart. J. Roy. Meteor. Soc., 137, 2174 2193, https://doi.org/10.1002/qj.891.

_ S. C. Jones, C. A. Davis, P. A. Harr, and M. Weissmann, 2013a: The impact of Typhoon Jangmi (2008) on the midlatitude flow. Part I: Upper-level ridgebuilding and modification of the jet. Quart. J. Roy. Meteor. Soc., 139, 2148-2164, https://doi.org/10.1002/qj.2091.

$\longrightarrow, \ldots$, and $-2013 \mathrm{~b}$ : The impact of Typhoon Jangmi (2008) on the midlatitude flow. Part II: Downstream evolution. Quart. J. Roy. Meteor. Soc., 139, 2148-2164, https://doi.org/ 10.1002/qj.2091.

— S. T. K. Lang, and J. H. Keller, 2015: A quantitative assessment of the sensitivity of the downstream midlatitude flow response to extratropical transition of tropical cyclones. Geophys. Res. Lett., 42, 9521-9529, https://doi.org/10.1002/ 2015GL065764.

Hakim, G. J., 2003: Developing wave packets in the North Pacific storm track. Mon. Wea. Rev., 131, 2824-2837, https://doi.org/ 10.1175/1520-0493(2003)131<2824:DWPITN>2.0.CO;2.

Harr, P. A., and J. M. Dea, 2009: Downstream development associated with the extratropical transition of tropical cyclones over the western North Pacific. Mon. Wea. Rev., 137, 12951319, https://doi.org/10.1175/2008MWR2558.1.

— D. D. Anwender, and S. C. Jones, 2008: Predictability associated with the downstream impacts of the extratropical transition of tropical cyclones: Methodology and a case study of Typhoon Nabi (2005). Mon. Wea. Rev., 136, 3205-3225, https://doi.org/ 10.1175/2008MWR2248.1.

Hodur, R. M., 1997: The Naval Research Laboratory's Coupled Ocean/Atmosphere Mesoscale Prediction System (COAMPS). Mon. Wea. Rev., 125, 1414-1430, https://doi.org/10.1175/15200493(1997) $125<1414$ :TNRLSC $>2$.0.CO;2.

Hodyss, D., and E. Hendricks, 2010: The resonant excitation of baroclinic waves by the divergent circulation of recurving tropical cyclones. J. Atmos. Sci., 67, 3600-3616, https://doi.org/ 10.1175/2010JAS3459.1.

Hoskins, B. J., and T. Ambrizzi, 1993: Rossby wave propagation on a realistic longitudinally varying flow. J. Atmos. Sci., 50, 1661-1671, https://doi.org/10.1175/1520-0469(1993)050<1661: RWPOAR $>2.0 . \mathrm{CO} ; 2$.

Jin, Y., W. T. Thompson, S. Wang, and C.-S. Liou, 2007: A numerical study of the effect of dissipative heating on tropical cyclone intensity. Wea. Forecasting, 22, 950-966, https://doi.org/ 10.1175/WAF1028.1.

Kain, J. S., 2004: The Kain-Fritsch convective parameterization: An update. J. Appl. Meteor., 43, 170-181, https://doi.org/10.1175/ 1520-0450(2004)043<0170:TKCPAU > 2.0.CO;2.

Keller, J. H., 2017: Amplification of the downstream wave train during extratropical transition: Sensitivity studies. Mon. Wea. Rev., 145, 1529-1548, https://doi.org/10.1175/MWR-D16-0193.1.

_- and Coauthors, 2019: The extratropical transition of tropical cyclones Part II: Interaction with the midlatitude flow, downstream impacts, and implications for predictability. Mon. Wea. Rev., 147, 1077-1106, https://doi.org/10.1175/ MWR-D-17-0329.1.

Komaromi, W. A., and J. D. Doyle, 2018: On the dynamics of tropical cyclone and trough interactions. J. Atmos. Sci., 75, 2687-2709, https://doi.org/10.1175/JAS-D-17-0272.1.

Lillo, S. P., and D. B. Parsons, 2017: Investigating the dynamics of error growth in ECMWF medium-range forecast busts. Quart. J. Roy. Meteor. Soc., 143, 1211-1226, https://doi.org/10.1002/qj.2938.

Lin, Y.-L., R. D. Farley, and H. D. Orville, 1983: Bulk parameterization of the snow field in a cloud model. J. Climate Appl. Meteor., 22, 1065-1092, https://doi.org/10.1175/1520-0450(1983) 022<1065:BPOTSF $>2.0 . \mathrm{CO} ; 2$.

Lindzen, R. S., and B. Farrell, 1980: A simple approximate result for the maximum growth rate of baroclinic instabilities. J. Atmos. Sci., 37, 1648-1654, https://doi.org/10.1175/15200469(1980)037<1648:ASARFT>2.0.CO;2.

Manola, I., F. Selten, H. De Vries, and W. Hazeleger, 2013: "Waveguidability" of idealized jets. J. Geophys. Res. Atmos., 118, 10 432-10 440, https://doi.org/10.1002/jgrd.50758.

Martínez-Alvarado, O., E. Madonna, S. L. Gray, and H. Joos, 2016: A route to systematic error in forecasts of Rossby waves. Quart. J. Roy. Meteor. Soc., 142, 196-210, https://doi.org/ 10.1002/qj.2645.

Martius, O., C. Schwierz, and H. C. Davies, 2010: Tropopause-level waveguides. J. Atmos. Sci., 67, 866-879, https://doi.org/ 10.1175/2009JAS2995.1.

Nolan, D. S., M. T. Montgomery, and L. D. Grasso, 2001: The wavenumber one instability and trochoidal motion of hurricanelike vortices. J. Atmos. Sci., 58, 3243-3270, https://doi.org/ 10.1175/1520-0469(2001)058<3243:TWOIAT $>2.0$. CO;2.

Orlanski, I., and J. P. Sheldon, 1995: Stages in the energetics of baroclinic systems. Tellus, 47, 605-628, https://doi.org/10.3402/ tellusa.v47i5.11553.

Pantillon, F., J. P. Chaboureau, C. Lac, and P. Mascart, 2013: On the role of a Rossby wave train during the extratropical transition of Hurricane Helene (2006). Quart. J. Roy. Meteor. Soc., 139, 370-386, https://doi.org/10.1002/qj.1974.

Postel, G. A., and M. H. Hitchman, 1999: A climatology of Rossby wave breaking along the subtropical tropopause. J. Atmos. Sci., 56, 359-373, https://doi.org/10.1175/1520-0469(1999) 056<0359:ACORWB > 2.0.CO;2.

Quinting, J. F., and S. C. Jones, 2016: On the impact of tropical cyclones on Rossby wave packets: A climatological perspective. Mon. Wea. Rev., 144, 2021-2048, https://doi.org/10.1175/ MWR-D-14-00298.1.

Reynolds, C. A., M. S. Peng, and J.-H. Chen, 2009: Recurving Tropical Cyclones: Singular Vector Sensitivity and Downstream Impacts. Mon. Wea. Rev., 137, 1320-1337, https:// doi.org/10.1175/2008MWR2652.1.

Riboldi, J., M. Röthlisberger, and C. M. Grams, 2018: Rossby wave initiation by recurving tropical cyclones in the western North Pacific. Mon. Wea. Rev., 146, 1283-1301, https://doi.org/ 10.1175/MWR-D-17-0219.1.

_ C. M. Grams, M. Riemer, and H. M. Archambault, 2019: A phase locking perspective on Rossby wave amplification and atmospheric blocking downstream of recurving western North Pacific tropical cyclones. Mon. Wea. Rev., 147, 567-589, https://doi.org/10.1175/MWR-D-18-0271.1.

Riemer, M., and S. C. Jones, 2010: The downstream impact of tropical cyclones on a developing baroclinic wave in idealized scenarios of extratropical transition. Quart. J. Roy. Meteor. Soc., 136, 617-637, https://doi.org/10.1002/qj.605. 
and - 2014: Interaction of a tropical cyclone with a high-amplitude, midlatitude wave pattern: Waviness analysis, trough deformation and track bifurcation. Quart. J. Roy. Meteor. Soc., 140, 1362-1376, https://doi.org/10.1002/qj.2221.

- — - and C. A. Davis, 2008: The impact of extratropical transition on the downstream flow: An idealized modelling study with a straight jet. Quart. J. Roy. Meteor. Soc., 134, 6991, https://doi.org/10.1002/qj.189.

Ritchie, E. A., and R. L. Elsberry, 2003: Simulations of the extratropical transition of tropical cyclones: Contributions by the midlatitude upper-level trough to reintensification. Mon. Wea. Rev., 131, 2112-2128, https://doi.org/10.1175/ 1520-0493(2003)131<2112:SOTETO > 2.0.CO;2.

$\longrightarrow$, and _ 2007: Simulations of the extratropical transition of tropical cyclones: Phasing between the upper-level trough and tropical cyclones. Mon. Wea. Rev., 135, 862-876, https:// doi.org/10.1175/MWR3303.1.

—, and W. M. Frank, 2007: Interactions between simulated tropical cyclones and an environment with a variable Coriolis parameter. Mon. Wea. Rev., 135, 1889-1905, https://doi.org/ 10.1175/MWR3359.1.

Rodwell, M. J., and Coauthors, 2013: Characteristics of occasional poor medium-range weather forecasts for Europe. Bull. Amer. Meteor. Soc., 94, 1393-1405, https://doi.org/10.1175/BAMS-D12-00099.1.

Rutledge, S. A., and P. V. Hobbs, 1983: The mesoscale and microscale structure and organization of clouds and precipitation in midlatitude cyclones. VIII: A model for the "seederfeeder" process in warm-frontal rainbands. J. Atmos. Sci., 40 , 1185-1206, https://doi.org/10.1175/1520-0469(1983)040<1185: TMAMSA $>2.0 . \mathrm{CO} ; 2$.

Scheck, L., S. C. Jones, and M. Juckes, 2011: The resonant interaction of a tropical cyclone and a tropopause front in a barotropic model. Part I: Zonally oriented front. J. Atmos. Sci., 68, 405-419, https://doi.org/10.1175/2010JAS3482.1.

Schwierz, C., S. Dirren, and H. C. Davies, 2004: Forced waves on a zonally aligned jet stream. J. Atmos. Sci., 61, 73-87,
https://doi.org/10.1175/1520-0469(2004)061<0073:FWOAZA > 2.0.CO;2.

Simmons, A. J., and B. J. Hoskins, 1979: The downstream and upstream development of unstable baroclinic waves. J. Atmos. Sci., 36, 1239-1254, https://doi.org/10.1175/1520-0469(1979) 036<1239:TDAUDO $>2.0 . \mathrm{CO} ; 2$.

Torn, R. D., 2010: Diagnosis of the downstream ridging associated with extratropical transition using short-term ensemble forecasts. J. Atmos. Sci., 67, 817-833, https://doi.org/10.1175/ 2009JAS3093.1.

_, 2017: A comparison of the downstream predictability associated with ET and baroclinic cyclones. Mon. Wea. Rev., 145, 4651-4672, https://doi.org/10.1175/MWR-D-17-0083.1.

, and G. J. Hakim, 2015: Comparison of wave packets associated with extratropical transition and winter cyclones. Mon. Wea. Rev., 143, 1782-1803, https://doi.org/10.1175/MWR-D14-00006.1.

Wang, Y., and G. J. Holland, 1996: The beta drift of baroclinic vortices. Part II: Diabatic vortices. J. Atmos. Sci., 53, 3737-3756, https://doi.org/10.1175/1520-0469(1996)053<3737: TBDOBV $>2.0 . \mathrm{CO} ; 2$.

Wirth, V., M. Riemer, E. K. M. Chang, and O. Martius, 2018: Rossby Wave Packets on the Midlatitude Waveguide-A review. Mon. Wea. Rev., 146, 1965-2001, https://doi.org/ 10.1175/MWR-D-16-0483.1.

Wu, C.-C., and K. A. Emanuel, 1993: Interaction of a baroclinic vortex with background shear: Application to hurricane movement. J. Atmos. Sci., 50, 62-76, https://doi.org/10.1175/ 1520-0469(1993)050<0062:IOABVW $>2.0 . \mathrm{CO} ; 2$.

- and _ 1994: On hurricane outflow structure. J. Atmos. Sci., 51, 1995-2003, https://doi.org/10.1175/1520-0469(1994) 051<1995:OHOS > 2.0.CO;2.

, and Y. Kurihara, 1996: A numerical study of the feedback mechanisms of hurricane-environment interaction on hurricane movement from the potential vorticity perspective. J. Atmos. Sci., 53, 2264-2282, https://doi.org/10.1175/15200469(1996)053<2264:ANSOTF>2.0.CO;2. 